\title{
Risk of rheumatic disease in breast implant users: a qualitative systematic review
}

\author{
Sabrina Hoa ${ }^{1,2}$, Kathleen Milord ${ }^{1}$, Marie Hudson ${ }^{3}$, Stephen C. Nicolaidis ${ }^{1,4}$, Josiane Bourré-Tessier ${ }^{1,2}$ \\ ${ }^{1}$ Faculty of Medicine, Université de Montréal, Montreal, Quebec, Canada; ${ }^{2}$ Division of Rheumatology, Department of Medicine, Centre hospitalier \\ de l'Université de Montréal, Université de Montréal, Montreal, Quebec, Canada; ${ }^{3}$ Division of Rheumatology, Department of Medicine, Jewish \\ General Hospital, McGill University, Montreal, Quebec, Canada; ${ }^{4}$ Division of Plastic Surgery, Department of Surgery, Centre hospitalier de \\ l’Université de Montréal, Université de Montréal, Montreal, Quebec, Canada \\ Contributions: (I) Conception and design: S Hoa, K Milord, M Hudson, J Bourré-Tessier; (II) Administrative support: S Hoa; (III) Provision of study \\ materials: S Hoa, K Milord; (IV) Collection and assembly of data: S Hoa, K Milord; (V) Data analysis and interpretation: S Hoa, K Milord, M \\ Hudson, SC Nicolaidis, J Bourré-Tessier; (VI) Manuscript writing: All authors; Final approval of manuscript: All authors. \\ Correspondence to: Dr. Sabrina Hoa. Centre hospitalier de l'Université de Montréal, 1051 Sanguinet, Pavilion B-5, Montreal, Quebec, H2X 3E4, \\ Canada. Email: sabrina.hoa@mail.mcgill.ca.
}

Background: Recent studies on the risk of rheumatic disease among breast implant users have reported conflicting results. The primary objective of this study was to provide a systematic and critical review of the literature on the association between breast implants and the risk of rheumatic disease.

Methods: A qualitative systematic review was conducted in PubMed, MEDLINE, EMBASE, EBM-Reviews and CINAHL Complete from database inception to June 23rd, 2021. Eligible papers were full-length articles in English or French reporting original data on the incident risk of rheumatic disease among individuals with and without breast implants. Data were extracted from published reports and appraised using the Newcastle-Ottawa scale. The main outcome was incident risk of systemic sclerosis (SSc), Sjögren's syndrome (SS), systemic lupus erythematosus (SLE), rheumatoid arthritis (RA), fibromyalgia and other rheumatic disorders and symptoms.

Results: Out of 3,425 identified citations, 86 met inclusion criteria. Two cohort studies suggested a twofold increase in risk of SSc, whereas three case-control studies showed no increase in risk. Three cohort studies did not find an increased risk of incident and confirmed SS among breast implant users, however symptoms of sicca, myalgia and fatigue were reported more frequently. A meta-analysis of heterogenous studies reported a less than two-fold increase in risk of RA. Studies did not support an association with SLE. Insufficient evidence was available for autoimmune myositis and other rheumatic diseases. Implant rupture detected on imaging was not clearly associated with incident rheumatic disease, although no studies specifically examined the risk associated with acute/traumatic rupture. Little data was available on the safety of saline breast implants. Explantation often led to temporary improvement.

Conclusions: Based on a small number of high-quality and methodologically robust studies, an association between breast implants and a small increase in risk of SSc and RA could not be excluded. Symptoms of sicca, myalgia and fatigue were reported more frequently among breast implant users. Overall, there remains much uncertainty in regard to the association between breast implants and the risk of incident rheumatic diseases. Individuals considering the placement of breast implants should be informed of this uncertainty.

Trial Registration: This study was registered in the PROSPERO database (\#CRD42019133616).

Keywords: Breast implant; silicone; rheumatic diseases; systematic review

Submitted Apr 21, 2021. Accepted for publication Jul 23, 2021.

doi: 10.21037 /gs-21-266

View this article at: https://dx.doi.org/10.21037/gs-21-266

\footnotetext{
$\wedge$ ORCID: 0000-0002-9466-5729.
} 


\section{Introduction}

Breast implants have been used for cosmetic and reconstructive purposes since its introduction in 1962. Multiple studies have since raised concerns relating to their safety, with conflicting reports linking breast implants to systemic autoimmune diseases. Silicone contained within breast implants or as part of the capsules has been hypothesized to induce autoimmunity through an adjuvant effect in genetically predisposed individuals. Given growing concerns over their safety, the United States Food and Drug Administration (FDA) imposed a moratorium on silicone breast implants in 1992. This moratorium, which eventually extended worldwide, was lifted in 2006 based on a lack of evidence of harm, and large post-approval studies were launched to monitor the long-term safety of breast implants. In 2008, an association between macrotextured breast implants and a rare T-cell lymphoma was identified (1) and later designated by the World Health Organization as the "breast implant-associated anaplastic large cell lymphoma" in 2016. This finding contributed to renewed questions on the general safety of breast implants by patients, the general public and the media, including in regard to rheumatic diseases.

Conflicting results from post-approval studies $(2,3)$ and other large cohort studies have since been published, with some reporting large increases in risk of connective tissue diseases (CTDs), while others finding no association. In September 2020, the FDA published recommendations to incorporate the "breast implant illness" (encompassing symptoms of joint pain, fatigue, memory loss, "brain fog" and rash) as part of the informed consent form and as a boxed warning for all types of breast implants (4). In the midst of this debate, algorithms for screening and management of rheumatic disease in the context of breast implants were published, recommending pre- and postoperative screening for rheumatic diseases, with the rheumatologist being given a key role to recommend for or against breast implant placement and removal $(5,6)$.

In response to increasing demands for a rheumatologic opinion on the role of breast implants in the risk and management of rheumatic diseases, we undertook a systematic, critical and up-to-date review of the literature on the association between breast implants and rheumatic diseases. The primary objective was to determine whether individuals exposed to breast implants have an increased risk of incident rheumatic disease. Secondary objectives were to explore predictors and markers of rheumatic disease and to report on the course of rheumatic disease after explantation. We present the following article in accordance with the PRISMA reporting checklist (available at https://dx.doi. org/10.21037/gs-21-266).

\section{Methods}

\section{Data sources and search strategy}

This systematic review was conducted using a predefined protocol registered in the PROSPERO database (\#CRD42019133616). Five health-related databases with international coverage (PubMed, MEDLINE, EMBASE, EBM-Reviews and CINAHL Complete) and grey literature (Supplementary File 1: https://cdn.amegroups.cn/static/ public/gs-21-266-1.pdf) were searched from database inception to May $2^{\text {nd }}, 2019$. No limit on date, language or study type was placed on the initial database search. The complete search (Supplementary File 1: https://cdn. amegroups.cn/static/public/gs-21-266-1.pdf) was developed with the assistance of a professional librarian. References of selected papers were hand-searched to identify additional relevant studies. Records were imported into an EndNote database to facilitate removal of duplicates and article screening. Final database search was conducted on June $23^{\text {rd }}, 2021$.

\section{Eligibility criteria}

We included published, full-length manuscripts reporting original data (including meta-analyses, randomized controlled trials, prospective and retrospective cohort studies, case-control studies, cross-sectional studies and case series) on human subjects in English or French language. Due to the high risk of selection bias in case reports, we selected studies that reported on at least 20 or more study patients. Subjects of any gender could be included. For the primary objective, the risk of incident rheumatic disease was compared among individuals with and without breast implants of any type. The main outcomes were incident risks of systemic sclerosis (SSc), Sjögren's syndrome (SS), rheumatoid arthritis (RA), systemic lupus erythematosus (SLE), autoimmune myositis, mixed CTD (MCTD), undifferentiated CTD (UCTD), vasculitis, spondyloarthropathies, sarcoidosis, fibromyalgia and chronic fatigue syndrome (CFS). In addition, the incident risk of rheumatic symptoms, such as Raynaud's phenomenon, arthralgias, myalgias and sicca, was also examined. For 
the secondary objectives, the risk of rheumatic disease was compared according to the presence of predictors or markers, among subjects with breast implants.

\section{Study selection}

Two independent reviewers (SH, KM) screened titles and abstracts. Titles for which an abstract was unavailable or for which the decision was uncertain were included for fulltext review. Any disagreement was resolved by consensus. Two reviewers (SH, KM) screened full-text papers for final selection and extracted data from published reports. Fulltext papers that could not be obtained through institutional holdings were requested through interlibrary loans. All papers not meeting eligibility criteria were excluded and reason for exclusion was noted. If more than one study was published with the same cohort, we retained the publication with the largest sample.

\section{Data extraction and data items}

Data extraction was performed using a pre-piloted form to collect characteristics of studies, populations (source, selection criteria), breast implants (type, duration, indication, complications), outcomes (definitions, ascertainment, temporality), outcome measures [odds ratios (OR), relative risks (RR), hazard ratios (HR) and standardized incidence ratios (SIR) with $95 \%$ confidence intervals (CIs), confounding variables used for adjustments, $\%$ improvement after explantation] and funding sources. Investigators communicated with study authors to obtain additional data as needed.

\section{Risk of bias assessment}

Quality of cohort and case-control studies were assessed using the Newcastle-Ottawa Scale (NOS) (7). The maximum score was nine (four points for selection, two points for comparability and three points for outcome categories). Cross-sectional studies were evaluated using the same criteria, with the understanding that the lack of follow-up automatically reduced the score. Studies with scores of six and above were classified as "high quality".

\section{Statistical analysis}

In this qualitative systematic review, data were summarized in tabular form by rheumatic disease, stratified by comparator population and ordered by NOS and sample size. Descriptions of study features were tabulated next to risk estimates to facilitate critical appraisal. Investigators (SH, KM, JBT, MH, SCN) met in person on two occasions to review the data and resolved disagreements by consensus. Heterogeneity in study characteristics precluded further quantitative analyses (see Discussion).

\section{Results}

Search results and reasons for exclusion are summarized in Figure 1. The electronic search identified 3,425 potentially relevant citations. Five additional citations were identified through search of reference lists. After removal of duplicate papers $(n=1,401)$ and ineligible papers based on title and abstract $(\mathrm{n}=1,762)$ and full text review $(\mathrm{n}=181), 86$ studies were selected for inclusion. Among these, 46 studies addressed the risk of rheumatic disease; 7 additional studies reported on disease predictors; 18 studies reported on immunological markers; and 14 studies reported on disease course after explantation. The majority (56\%) were published from 1992 to 2000, 29\% were published from 2001 to 2010 and 14\% were published from 2011 to 2021 .

\section{Risk of CTDs}

\section{SSc and Raynaud's phenomenon}

Studies reporting on the risk of SSc are presented in Table 1 $(2,3,8-23)$. Although many of these studies were scored as "high-quality" based on the NOS, few presented results based on confirmed (rather than self-reported) and incident (rather than prevalent) diagnoses of SSc, within cohorts of sufficient sample size and with sufficient follow-up time to detect this rare disease with possibly latent onset. After excluding studies in which risk could not be estimated due to zero values, two cohort studies and three case-control studies met these criteria.

First, in a large Danish cohort of 2,761 women with cosmetic breast implants identified from a national hospital registry and from 8 private clinics, Fryzek et al. reported a HR of 1.7 (95\% CI: 0.4-7.7) for incident SSc compared to women with other cosmetic surgeries or breast reductions, and a SIR of 2.9 (95\% CI: 0.6-8.3) compared to the general population (8). Secondly, in a large Israeli cohort of 1,797 women with mostly cosmetic breast implants identified from a national healthcare database reported by Watad et al. (14), the prevalence OR was 1.63 (95\% CI: 1.26-2.11), but after excluding patients with any prevalent autoimmune 


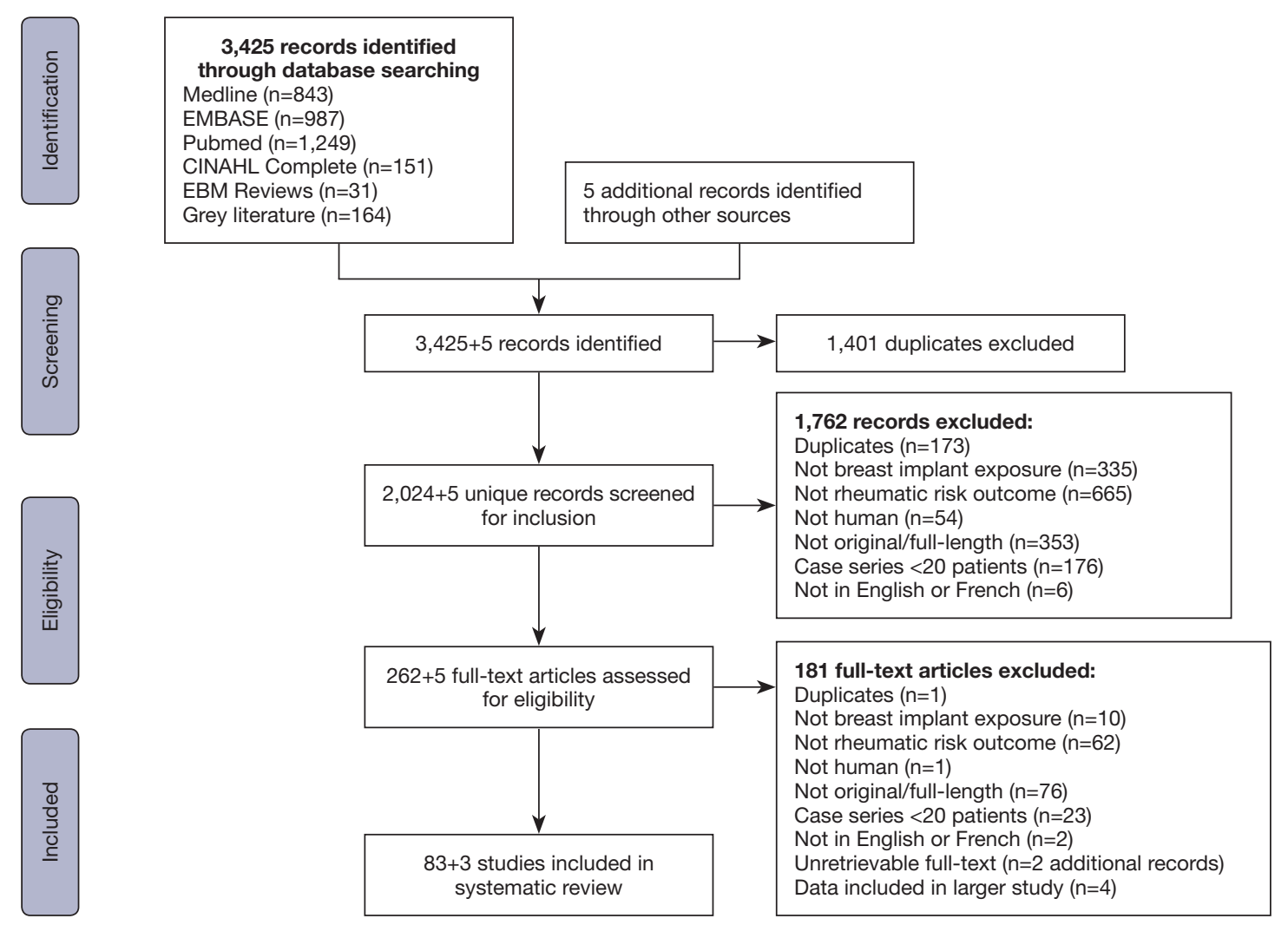

Figure 1 PRISMA flowchart of study selection process.

or rheumatic disease, the HR was 2.43 (95\% CI: 0.62-9.55) compared to women from the community (disease-specific HR obtained by personal communication). In the latter analysis, the time at risk was censored at the onset of any autoimmune or rheumatic disorder, such that the risk of SSc may have been underestimated if onset of another disorder (such as fibromyalgia or hypothyroidism) occurred prior to SSc diagnosis. Overall, these two cohort studies did not demonstrate a statistically significant increase in the risk of incident SSc, although CIs were wide, with point estimates suggesting an over two-fold risk increase. In addition, three high-quality case-control studies reported no increase in odds of breast implant exposure among SSc patients compared to community or general practice controls, with a combined OR of 1.02 (95\% CI: 0.56-1.84) $(20,22,24)$. Interestingly, two studies reported a higher frequency of past exposure to breast implants in SSc patients with antiRNA polymerase III autoantibodies (13-16\%) compared to SSc patients with anti-topoisomerase I $(0-0.6 \%)$ or anticentromere autoantibodies (1.0-1.2\%) $(25,26)$.

Furthermore, the risk of self-reported symptoms of
Raynaud's phenomenon (Supplementary File 2: https://cdn. amegroups.cn/static/public/gs-21-266-2.pdf) (9,10,12,15,27$35)$ was higher in patients with breast implants in three studies $(9,28,33)$, including a large study by Brinton et al. in which Raynaud's was reported in $1.3 \%$ of 7,234 women with cosmetic breast implants, compared to only $0.5 \%$ of women with other cosmetic surgeries (RR 2.6, 95\% CI: 1.3-5.1) (9). In contrast, the risk of Raynaud's phenomenon was not increased in five other smaller studies $(10,12,29-31)$.

\section{SS and sicca symptoms}

Three cohort studies reported on the risk of incident and confirmed diagnoses of SS (Table 2). In the Danish cohort by Fryzek et al., the risk of SS was not increased among women with cosmetic breast implants compared to other cosmetic surgeries or breast reductions (HR 1.3, 95\% CI: $0.3-7.2$ ) or compared to national rates (SIR 1.0, 95\% CI: 0.1-3.5) (8). In the Israeli cohort by Watad et al., breast implants were associated with an increased risk of prevalent SS (OR 1.58, 95\% CI: 1.26-1.97) (14), but after excluding patients with any prevalent autoimmune or rheumatic 
Table 1 Summary of studies reporting on the association between breast implants and risk of systemic sclerosis

\begin{tabular}{|c|c|c|c|c|c|c|c|c|c|}
\hline $\begin{array}{l}\text { Study (year) } \\
\text { (reference) }\end{array}$ & $\begin{array}{c}\% \text { in } \\
\text { silicone; } \\
\% \text { C }\end{array}$ & $\begin{array}{l}\text { Implant } \\
\text { group, } \mathrm{n} / \mathrm{N}^{*}\end{array}$ & $\begin{array}{l}\text { Comparator } \\
\text { group, } \mathrm{n} / \mathrm{N}^{\star}\end{array}$ & $\begin{array}{c}\text { Measure of } \\
\text { effect }(95 \% \mathrm{Cl})\end{array}$ & $\begin{array}{l}\text { Follow-up, } \\
\text { years }\end{array}$ & Adjustments & Notes & NOS & $\$$ \\
\hline
\end{tabular}

\begin{tabular}{|c|c|c|c|c|c|c|c|c|}
\hline $\begin{array}{l}\text { Fryzek et al. } \\
(2007)(8)\end{array}$ & $\begin{array}{l}>84 \% \\
100 \% \text { C }\end{array}$ & $3 / 2,761$ & $5 / 8,807$ & HR $1.7(0.4-7.7)$ & 13.4 & $\begin{array}{l}\text { Age, sex, calendar } \\
\text { year, clinic, time } \\
\text { since operation }\end{array}$ & $\begin{array}{l}\text { Danish national } \\
\text { hospital registry + } \\
\text { private clinics }\end{array}$ & 9 \\
\hline
\end{tabular}

Cohort studies comparing with other cosmetic surgeries

\begin{tabular}{|c|c|c|c|c|c|c|c|c|c|}
\hline \multirow[t]{2}{*}{$\begin{array}{l}\text { Brinton et al. } \\
(2004)(9)\end{array}$} & $\begin{array}{c}50 \% \\
100 \% \mathrm{C}\end{array}$ & $2 / 7,234$ & $0 / 2,138$ & $\begin{array}{l}\text { RR NR (0.4-NR) } \\
\text { (confirmed) }\end{array}$ & 12.1 & $\begin{array}{l}\text { Age, sex, race, } \\
\text { calendar period }\end{array}$ & $\begin{array}{l}18 \text { plastic surgery } \\
\text { practices }\end{array}$ & 9 & NR \\
\hline & & $23 / 7,234$ & $3 / 2,138$ & $\begin{array}{c}\text { RR } 3.0 \text { (0.8-10.9) } \\
\text { (self-reported) }\end{array}$ & & & & 8 & \\
\hline $\begin{array}{l}\text { Englert et al. } \\
(2001)(10)\end{array}$ & $\begin{array}{c}100 \% \\
100 \% \mathrm{C}\end{array}$ & $1 / 458$ & $1 / 687$ & $\begin{array}{c}\text { RR 1.50 } \\
(0.09-24.06)\end{array}$ & 15 & $\begin{array}{l}\text { Age, sex, clinic, } \\
\text { calendar year }\end{array}$ & $\begin{array}{c}16 \text { plastic surgery } \\
\text { practices }\end{array}$ & 9 & Yes \\
\hline $\begin{array}{l}\text { Edworthy et al. } \\
\text { (1998) (11) }\end{array}$ & $\begin{array}{c}71 \% ; \\
100 \% \mathrm{C}\end{array}$ & $0 / 1,576$ & $3 / 725$ & RR 0 & 12 & N/A & $\begin{array}{l}\text { Canadian provincial } \\
\text { health registry }\end{array}$ & 8 & No \\
\hline $\begin{array}{l}\text { Wells et al. } \\
\text { (1994) (12) }\end{array}$ & $\begin{array}{l}100 \% \\
\% \text { C NR }\end{array}$ & $0 / 222$ & $0 / 80$ & $\begin{array}{l}\text { RR not } \\
\text { calculable }\end{array}$ & $<5$ & $\mathrm{~N} / \mathrm{A}$ & $\begin{array}{c}\text { Single plastic } \\
\text { surgery practice }\end{array}$ & 6 & NR \\
\hline
\end{tabular}

Cohort studies comparing with breast reduction surgeries

$\begin{array}{lccccccc}\begin{array}{l}\text { Nyren et al. } \\ \text { (1998) (13) }\end{array} & 56 \% ; & 0 / 7,442 & 3 / 3,353 & \text { RR } 0 & 8.0 & \text { N/A } & \begin{array}{l}\text { Swedish national } \\ \text { inpatient registry }\end{array}\end{array}$

Cohort studies comparing with women from the community without breast implants

\begin{tabular}{|c|c|c|c|c|c|c|c|c|c|}
\hline \multirow[t]{2}{*}{$\begin{array}{l}\text { Watad et al. } \\
(2018)(14)\end{array}$} & $\begin{array}{l}100 \% \\
95 \% \text { C }\end{array}$ & $101 / 24,651$ & $242 / 98,604$ & $\begin{array}{c}\text { OR } 1.63(1.26-2.11) \\
\text { (prevalence) }\end{array}$ & \multirow[t]{2}{*}{9.7} & \multirow{2}{*}{$\begin{array}{c}\text { Age, sex, SES, } \\
\text { smoking, breast } \\
\text { cancer }\end{array}$} & \multirow[t]{2}{*}{$\begin{array}{l}\text { Israeli healthcare } \\
\text { database }\end{array}$} & 8 & \multirow[t]{2}{*}{ No } \\
\hline & & $3 / 1,797$ & $7 / 7,109$ & $\begin{array}{c}\text { HR } 2.43(0.62-9.55) \\
\quad \text { (incidence) }{ }^{\star}\end{array}$ & & & & 9 & \\
\hline $\begin{array}{l}\text { Gabriel et al. } \\
\text { (1994) (15) }\end{array}$ & $\begin{array}{l}85 \% \\
71 \% \mathrm{C}\end{array}$ & $0 / 749$ & $1 / 1,498$ & RR 0 & 7.8 & $\mathrm{~N} / \mathrm{A}$ & $\begin{array}{l}\text { Tertiary care and } \\
\text { affiliated centers }\end{array}$ & 8 & No \\
\hline \multicolumn{10}{|c|}{ Cohort studies comparing with female health professionals without breast implants } \\
\hline $\begin{array}{l}\text { Sanchez- } \\
\text { Guerrero et al. } \\
\text { (1995) (16) }\end{array}$ & $\begin{array}{l}74 \% \\
50 \% \text { C }\end{array}$ & $0 / 1,183$ & $14 / 86,318$ & RR 0 & 9.9 & $\mathrm{~N} / \mathrm{A}$ & $\begin{array}{l}\text { Nurses' Health } \\
\text { Study }\end{array}$ & 7 & Yes \\
\hline $\begin{array}{l}\text { Hennekens } \\
\text { et al. (1996) (17) }\end{array}$ & $\begin{array}{c}\text { NR; \% C } \\
\text { NR }\end{array}$ & $10 / 10,830$ & $314 / 384,713$ & $\begin{array}{c}3 \mathrm{HR} 1.84 \text { (0.98-3.46) } \\
\text { (self-reported) }\end{array}$ & $<4-\geq 10$ & $\begin{array}{l}\text { Age, sex, calendar } \\
\text { year, cancer, } \\
\text { implant duration }\end{array}$ & $\begin{array}{c}\text { Women's Health } \\
\text { Study }\end{array}$ & 6 & Yes \\
\hline $\begin{array}{l}\text { Lee et al. (2011) } \\
\text { (18) }\end{array}$ & $\begin{array}{l}70 \% \\
68 \% \mathrm{C}\end{array}$ & $1 / 3,950$ & $4 / 19,897$ & HR NR & 3.6 & N/A & $\begin{array}{c}\text { Women's Health } \\
\text { Study }\end{array}$ & 5 & \\
\hline
\end{tabular}

Cohort studies comparing with post-mastectomy reconstructive surgeries without implants

$\begin{array}{lccccccc}\begin{array}{l}\text { Greenland } \\ \text { et al. (2000) (19) NR; \% C }\end{array} & \text { NR } & \begin{array}{c}1 / 31,820 \\ \text { person-y }\end{array} & \text { NR } & \begin{array}{c}\text { RR 1.56 (0.34-7.08) } \\ \text { for SSc/myositis/ } \\ \text { fibrosclerosis }\end{array} & \begin{array}{c}\text { Limited } \\ \text { since surgery }\end{array} & \begin{array}{c}\text { Age, sex, time } \\ \text { prevalent not } \\ \text { excluded }\end{array}\end{array}$

Cohort studies comparing with national rates

\begin{tabular}{lccccccc}
$\begin{array}{l}\text { Fryzek et al. } \\
(2007)(8)\end{array}$ & $>84 \% ;$ & $3 / 2,761$ & NR & SIR $2.9(0.6-8.3)$ & 13.4 & $\begin{array}{c}\text { Age, sex, calendar } \\
\text { period }\end{array}$ & $\begin{array}{c}\text { Danish national } \\
\text { hospital registry }+ \\
\text { private clinics }\end{array}$ \\
\hline
\end{tabular}

Table 1 (continued) 
Table 1 (continued)

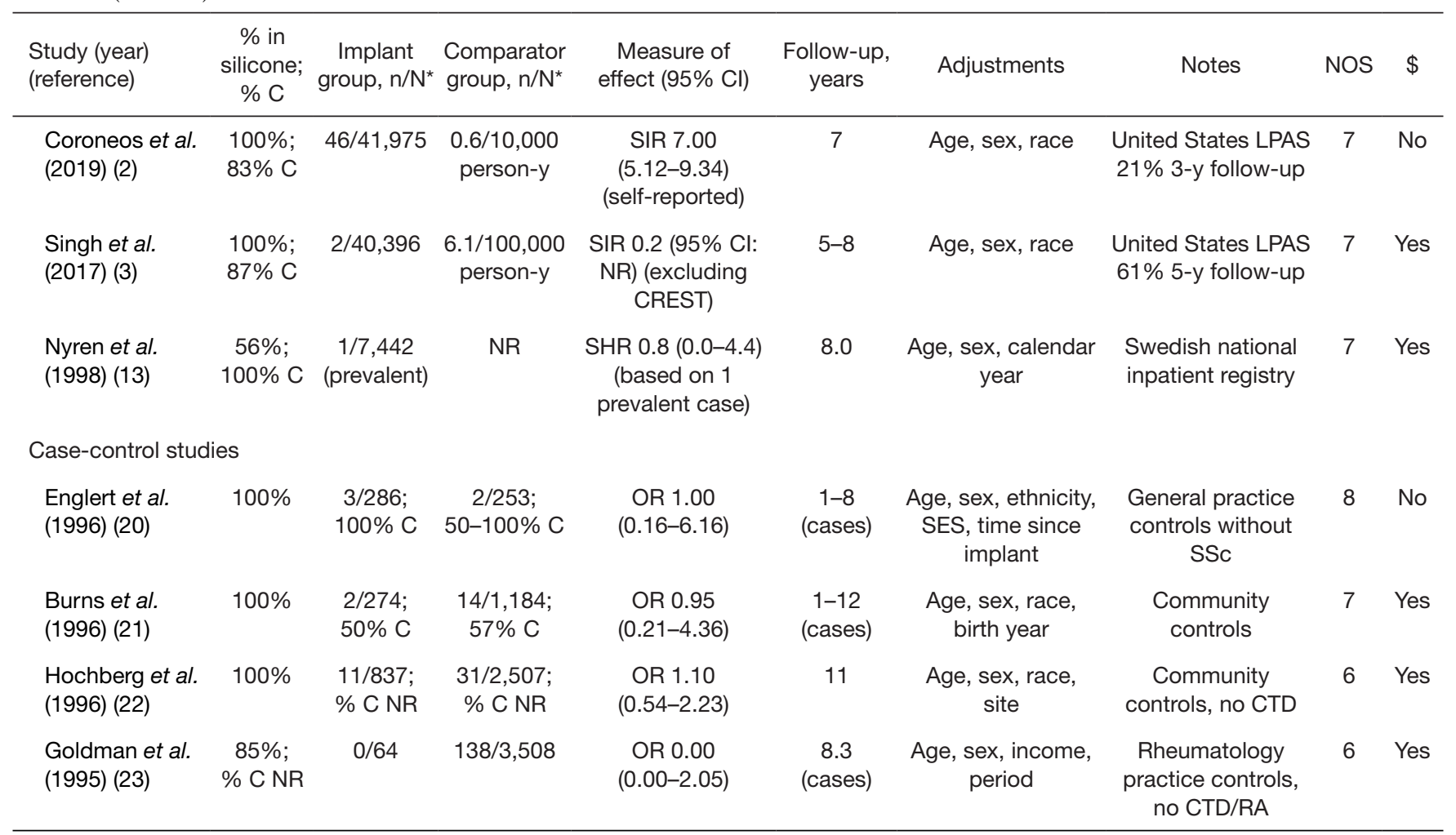

*, for case-control studies, case group (n breast implants/N); control group (n breast implants/N). In Watad et al., disease-specific HRs were obtained by personal communication. $\mathrm{C}$, cosmetic augmentation; $\mathrm{Cl}$, confidence interval; CTD, connective tissue disease; HR, hazard ratio; LPAS, long-term post-approval study; N/A, not applicable; NOS, Newcastle-Ottawa scale; NR, not reported; OR, odds ratio; RA, rheumatoid arthritis; RR, relative risk; SES, socioeconomic status; SHR, standardized hospitalization ratio; SIR, standardized incidence ratio; SSc, systemic sclerosis; $y$, years; \$, potential financial or other conflict of interest.

disease, the HR was 1.11 (95\% CI: 0.22-5.50) (personal communication) among women with breast implants compared to women from the community. Once again, this risk may have been underestimated if onset of another autoimmune or rheumatic disorder preceded the diagnosis of SS. Finally, in a Canadian cohort of 1,576 women with cosmetic breast implants identified from a provincial health registry, the risk of SS was not increased compared to 725 women undergoing other cosmetic surgeries (RR 0.99, 95\% CI: 0.17-5.94) (11).

Furthermore, four cohort studies from the United States evaluated the risk of incident but self-reported diagnoses of SS. In the study by Brinton et al., the risk of SS was much higher among 7,234 patients with cosmetic breast implants identified from 18 plastic surgery practices, compared to 2,138 patients with other types of plastic surgeries (RR 11.7, 95\% CI: 2.5-54.9) (9). However, when the diagnosis was reviewed by two rheumatologists, less than half of self-reported SS were deemed likely to represent SS (9). In addition, in the Women's Health Study, a retrospective study by Hennekens et al. found a HR of 1.49 (95\% CI: $0.97-2.28)$ for self-reported diagnoses of SS among 10,830 female health professionals with breast implants (17), and a prospective study by Lee $e t$ al. reported a HR of 2.78 (95\% CI: 1.29-5.98) for diagnoses of SS based on a validated patient questionnaire among 3,950 female health professionals with breast implants (18). Finally, in the large prospective study by Coroneos et al., which followed 41,975 patients with breast implants, the SIR for self-reported SS was 8.14 (95\% CI: 6.24-10.44) over 7 years of followup, albeit with significant attrition rates, with only $21 \%$ of patients being followed at 3 years (2).

In addition, three high-quality cohort studies found an increased risk in sicca symptoms among breast implant users compared to those with breast reduction surgeries (OR 2.2-2.5, $\mathrm{P}<0.05$ for sand or gravel sensation in the eyes) (28), 
Table 2 Summary of studies reporting on the association between breast implants and risk of Sjögren's syndrome

\begin{tabular}{|c|c|c|c|c|c|c|c|c|c|}
\hline $\begin{array}{l}\text { Study (year) } \\
\text { (reference) }\end{array}$ & $\begin{array}{c}\% \text { in } \\
\text { silicone; } \\
\% \text { C }\end{array}$ & $\begin{array}{c}\text { Implant } \\
\text { group, n/N* }\end{array}$ & $\begin{array}{l}\text { Comparator } \\
\text { group, } \mathrm{n} / \mathrm{N}^{*}\end{array}$ & $\begin{array}{l}\text { Measure of effect } \\
\qquad(95 \% \mathrm{Cl})\end{array}$ & $\begin{array}{c}\text { Follow-up, } \\
\text { years }\end{array}$ & Adjustments & Notes & NOS & $\$$ \\
\hline
\end{tabular}

Cohort studies comparing with other cosmetic surgeries and breast reduction surgeries

\begin{tabular}{|c|c|c|c|c|c|c|c|c|}
\hline $\begin{array}{l}\text { Fryzek et al. } \\
\text { (2007) (8) }\end{array}$ & $\begin{array}{l}>84 \% \\
100 \% \text { C }\end{array}$ & $2 / 2,761$ & $6 / 8,807$ & HR 1.3 (0.3-7.2) & 13.4 & $\begin{array}{l}\text { Age, sex, calendar } \\
\text { year, clinic, time } \\
\text { since operation }\end{array}$ & $\begin{array}{c}\text { Danish national } \\
\text { hospital registry + } \\
\text { private clinics }\end{array}$ & 9 \\
\hline
\end{tabular}

Cohort studies comparing with other cosmetic surgeries

\begin{tabular}{|c|c|c|c|c|c|c|c|c|c|}
\hline \multirow[t]{2}{*}{$\begin{array}{l}\text { Brinton et al. } \\
\text { (2004) (9) }\end{array}$} & $\begin{array}{c}50 \% \\
100 \% \text { C }\end{array}$ & $6 / 7,234$ & $0 / 2,138$ & $\begin{array}{l}\text { RR NR (0.4-NR) } \\
\quad \text { (confirmed) }\end{array}$ & 12.1 & $\begin{array}{l}\text { Age, sex, race, } \\
\text { calendar period }\end{array}$ & $\begin{array}{c}18 \text { plastic surgery } \\
\text { practices }\end{array}$ & 9 & NR \\
\hline & & $43 / 7,234$ & $2 / 2,138$ & $\begin{array}{l}\text { RR } 11.7 \text { (2.5-54.9) } \\
\quad \text { (self-reported) }\end{array}$ & & & & 8 & \\
\hline $\begin{array}{l}\text { Edworthy et al. } \\
\text { (1998) (11) }\end{array}$ & $\begin{array}{c}\text { 71\%; } \\
100 \% \text { C }\end{array}$ & $5 / 1,576$ & $4 / 725$ & RR 0.99 (0.17-5.94) & 12 & Age, sex & $\begin{array}{l}\text { Canadian } \\
\text { provincial health } \\
\text { registry }\end{array}$ & 8 & No \\
\hline
\end{tabular}

Cohort studies comparing with breast reduction surgeries

$\begin{array}{lccccccc}\text { Nyren et al. } & 56 \% ; & 1 / 7,442 & 0 / 3,353 & \text { RR not calculable } & 8.0 & \text { N/A } & \text { Swedish national } \\ \text { (1998) (13) } & 100 \% \text { C } & & & \text { Ynpatient registry }\end{array}$

Cohort studies comparing with women from the community without breast implants

\begin{tabular}{|c|c|c|c|c|c|c|c|c|c|}
\hline \multirow[t]{2}{*}{$\begin{array}{l}\text { Watad et al. } \\
(2018)(14)\end{array}$} & $\begin{array}{l}100 \% \\
95 \% \text { C }\end{array}$ & $123 / 24,651$ & $344 / 98,604$ & $\begin{array}{c}\text { OR } 1.58 \text { (1.26-1.97) } \\
\text { (prevalence) }\end{array}$ & \multirow[t]{2}{*}{9.7} & \multirow{2}{*}{$\begin{array}{l}\text { Age, sex, SES, } \\
\text { smoking, breast } \\
\text { cancer }\end{array}$} & \multirow[t]{2}{*}{$\begin{array}{c}\text { Israeli healthcare } \\
\text { database }\end{array}$} & 8 & \multirow[t]{2}{*}{ No } \\
\hline & & $2 / 1,797$ & $8 / 7,109$ & $\begin{array}{l}\text { HR } 1.11(0.22-5.50) \\
\quad \text { (incidence) }^{*}\end{array}$ & & & & 9 & \\
\hline $\begin{array}{l}\text { Gabriel et al. } \\
(1994)(15)\end{array}$ & $\begin{array}{l}85 \% \\
71 \% \mathrm{C}\end{array}$ & $1 / 749$ & $0 / 1,498$ & RR not calculable & 7.8 & N/A & $\begin{array}{l}\text { Tertiary care and } \\
\text { affiliated centers }\end{array}$ & 8 & No \\
\hline \multicolumn{10}{|c|}{ Cohort studies comparing with female health professionals without breast implants } \\
\hline $\begin{array}{l}\text { Sanchez- } \\
\text { Guerrero et al. } \\
(1995)(16)\end{array}$ & $\begin{array}{l}74 \% \\
50 \% \text { C }\end{array}$ & $0 / 1,183$ & 2/86,318 & RR 0 & 9.9 & N/A & $\begin{array}{l}\text { Nurses' Health } \\
\text { Study }\end{array}$ & 7 & Yes \\
\hline $\begin{array}{l}\text { Hennekens } \\
\text { et al. (1996) } \\
(17)\end{array}$ & $\begin{array}{l}\text { NR; \% C } \\
\quad \text { NR }\end{array}$ & $22 / 10,830$ & $752 / 384,713$ & $\begin{array}{l}\text { HR } 1.49 \text { (0.97-2.28) } \\
\quad \text { (self-reported) }\end{array}$ & $<4-\geq 10$ & $\begin{array}{l}\text { Age, sex, calendar } \\
\text { year, cancer, } \\
\text { implant duration }\end{array}$ & $\begin{array}{l}\text { Women's Health } \\
\text { Study }\end{array}$ & 6 & Yes \\
\hline $\begin{array}{l}\text { Lee et al. } \\
(2011)(18)\end{array}$ & $\begin{array}{l}70 \% \\
68 \% \text { C }\end{array}$ & $13 / 3,950$ & $25 / 19,897$ & $\begin{array}{l}\text { HR } 2.78 \text { (1.29-5.98) } \\
\text { (validated patient } \\
\text { questionnaire) }\end{array}$ & 3.6 & $\begin{array}{c}\text { Age, sex, body } \\
\text { mass index, } \\
\text { smoking, hormone, } \\
\text { cancer }\end{array}$ & $\begin{array}{l}\text { Women's Health } \\
\text { Study }\end{array}$ & 5 & Yes \\
\hline
\end{tabular}

Cohort studies comparing with post-mastectomy reconstructive surgeries without implants

\begin{tabular}{|c|c|c|c|c|c|c|c|}
\hline $\begin{array}{l}\text { Greenland } \\
\text { et al. (2000) } \\
\text { (19) }\end{array}$ & $\begin{array}{c}\text { NR; \% C } \\
\text { NR }\end{array}$ & $\begin{array}{l}6 / 31,820 \\
\text { person-y }\end{array}$ & NR & $\begin{array}{l}\text { RR } 2.21 \text { (1.00-4.93) } \\
\text { for sicca/Sjögren's }\end{array}$ & Limited & $\begin{array}{l}\text { Age, sex, time } \\
\text { since surgery }\end{array}$ & $\begin{array}{c}\text { Medicare (age } \\
\geq 65 \text { ); prevalent not } \\
\text { excluded }\end{array}$ \\
\hline
\end{tabular}

Cohort studies comparing with national rates

\begin{tabular}{|c|c|c|c|c|c|c|c|c|}
\hline $\begin{array}{l}\text { Fryzek et al. } \\
\text { (2007) (8) }\end{array}$ & $\begin{array}{l}>84 \% \\
100 \% \text { C }\end{array}$ & $2 / 2,761$ & NR & SIR $1.0(0.1-3.5)$ & 13.4 & $\begin{array}{c}\text { Age, sex, calendar } \\
\text { period }\end{array}$ & $\begin{array}{c}\text { Danish national } \\
\text { hospital registry + } \\
\text { private clinics }\end{array}$ & 9 \\
\hline
\end{tabular}

Table 2 (continued) 
Table 2 (continued)

\begin{tabular}{|c|c|c|c|c|c|c|c|c|c|}
\hline $\begin{array}{l}\text { Study (year) } \\
\text { (reference) }\end{array}$ & $\begin{array}{c}\% \text { in } \\
\text { silicone; } \\
\% \text { C }\end{array}$ & $\begin{array}{c}\text { Implant } \\
\text { group, } \mathrm{n} / \mathrm{N}^{*}\end{array}$ & $\begin{array}{l}\text { Comparator } \\
\text { group, } \mathrm{n} / \mathrm{N}^{*}\end{array}$ & $\begin{array}{l}\text { Measure of effect } \\
\qquad(95 \% \mathrm{Cl})\end{array}$ & $\begin{array}{l}\text { Follow-up, } \\
\text { years }\end{array}$ & Adjustments & Notes & NOS & $\$$ \\
\hline $\begin{array}{l}\text { Nyren et al. } \\
\text { (1998) (13) }\end{array}$ & $\begin{array}{c}56 \% \\
100 \% \text { C }\end{array}$ & $\begin{array}{c}3 / 7,442 \\
\text { (2 prevalent) }\end{array}$ & NR & $\begin{array}{l}\text { SHR } 1.8 \text { (0.4-5.4) } \\
\text { (mostly prevalent) }\end{array}$ & 8.0 & $\begin{array}{c}\text { Age, sex, calendar } \\
\text { year }\end{array}$ & $\begin{array}{l}\text { Swedish national } \\
\text { inpatient registry }\end{array}$ & 7 & Yes \\
\hline \multicolumn{10}{|c|}{ Case-control studies } \\
\hline $\begin{array}{l}\text { Goldman } \\
\text { et al. (1995) } \\
(23)\end{array}$ & $\begin{array}{c}85 \% ; \% \\
\text { C NR }\end{array}$ & $\begin{array}{c}2 / 49 \\
\text { (1 prevalent) }\end{array}$ & $138 / 3,508$ & OR 1.46 (0.36-6.39) & $\begin{array}{c}8.3 \\
\text { (cases) }\end{array}$ & $\begin{array}{l}\text { Age, sex, income, } \\
\text { period }\end{array}$ & $\begin{array}{c}\text { Rheumatology } \\
\text { practice controls, } \\
\text { no CTD or RA; FM } \\
\text { not excluded }\end{array}$ & 6 & Yes \\
\hline
\end{tabular}

*, for case-control studies, case group (n breast implants/N); control group (n breast implants/N). In Watad et al., disease-specific HRs were obtained by personal communication with study authors. C, cosmetic augmentation; $\mathrm{Cl}$, confidence interval; CTD, connective tissue disease; FM, fibromyalgia; HR, hazard ratio; N/A, not applicable; NOS, Newcastle-Ottawa scale; NR, not reported; OR, odds ratio; RA, rheumatoid arthritis; RR, relative risk; SES, socioeconomic status; SHR, standardized hospitalization ratio; SIR, standardized incidence ratio; y, years; \$, potential financial or other conflict of interest.

other cosmetic surgeries (OR 2.43, 95\% CI: 1.29-4.57 for regularly burning eyes) (31), or from the general population [OR 4.5, 95\% CI: 1.0-20.7 for dry mouth (29)]. Conversely, two high-quality cohort studies reported no increased risk in sicca symptoms compared to patients with other cosmetic surgeries (27) or from the community (15) (Supplementary File 2: https://cdn.amegroups.cn/static/ public/gs-21-266-2.pdf).

Overall, the above results are consistent with conclusions from the meta-analysis by Balk et al., which reported an increased risk of self-reported diagnoses of SS $(8.21,95 \%$ CI: 2.38-28.4), but not of confirmed diagnoses (1.26, $95 \%$ CI: 0.36-4.46) (36).

\section{RA and joint-related symptoms}

Nine cohort studies reported on the risk of incident and confirmed diagnoses of RA and found no significant increase in risk among women with breast implants compared to women with other cosmetic or breast reduction surgeries $(8-11,13)$ or from the community (14), to female health professionals $(16,18)$ or to national rates $(2,8)$ (Table 3) $(37,38)$. Average risk estimates mostly ranged from 1.3 to 1.9 with wide CIs. A meta-analysis by Balk et al. reported an overall effect size of 1.38 (95\% CI: 1.06-1.80) after combining mostly unadjusted and inadequately adjusted results from eleven studies with heterogeneous methodologies, including different comparator populations and studies with self-reported diagnoses (36). In addition, five studies reported on the incidence of joint-related symptoms (Supplementary File 2: https://cdn.amegroups. $\mathrm{cn} /$ static/public/gs-21-266-2.pdf). The risk of joint pain was increased in some studies $(28,31)$, but not others $(12,29)$. Two studies reported a 1.8 to 2.3 -fold increase in risk of morning or joint stiffness among women with breast implants compared to women with other cosmetic surgeries or from the general population $(15,29)$. Joint swelling was not more frequently reported $(12,27-29,31)$.

\section{SLE and related symptoms}

Five cohort studies with sufficient follow-up time found no association between breast implants and incident confirmed diagnoses of SLE, compared to women with other cosmetic surgeries (11) or breast reduction (13), to women from the community (14) or to national rates $(3,8)$ (Table 4) $(39,40)$. Meta-analyses also found no association with SLE $(36,41)$. The risk of photosensitivity was increased in one small study (31), but not in others $(15,28-30,34)$ (Supplementary File 2: https://cdn.amegroups.cn/static/public/gs-21-2662.pdf). Cohort studies reported no increased risk of malar or discoid rash (15), oral ulcers $(15,28,29,31)$, serositis or 
Table 3 Summary of studies reporting on the association between breast implants and risk of rheumatoid arthritis

\begin{tabular}{|c|c|c|c|c|c|c|c|c|c|}
\hline $\begin{array}{l}\text { Study (year) } \\
\text { (reference) }\end{array}$ & $\begin{array}{c}\% \text { in } \\
\text { silicone; } \\
\% \text { C }\end{array}$ & $\begin{array}{l}\text { Implant } \\
\text { group, } \mathrm{n} / \mathrm{N}^{*}\end{array}$ & $\begin{array}{l}\text { Comparator } \\
\text { group, } \mathrm{n} / \mathrm{N}^{*}\end{array}$ & $\begin{array}{l}\text { Measure of effect } \\
\qquad(95 \% \mathrm{Cl})\end{array}$ & $\begin{array}{l}\text { Follow-up, } \\
\text { years }\end{array}$ & Adjustments & Notes & NOS & $\$$ \\
\hline
\end{tabular}

Cohort studies comparing with other cosmetic surgeries and breast reduction surgeries

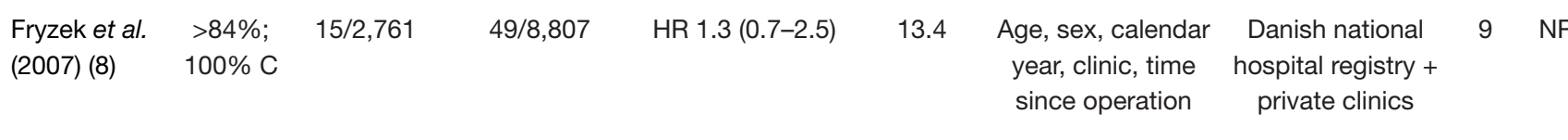

Cohort studies comparing with other cosmetic surgeries

\begin{tabular}{|c|c|c|c|c|c|c|c|c|c|}
\hline \multirow[t]{2}{*}{$\begin{array}{l}\text { Brinton et al. } \\
(2004)(9)\end{array}$} & $\begin{array}{c}50 \% \\
100 \% \mathrm{C}\end{array}$ & $16 / 7,234$ & $4 / 2,138$ & $\begin{array}{l}\text { RR } 1.3 \text { (0.5-3.8) } \\
\text { (confirmed) }\end{array}$ & 12.1 & $\begin{array}{l}\text { Age, sex, race, } \\
\text { calendar period }\end{array}$ & $\begin{array}{l}18 \text { plastic surgery } \\
\text { practices }\end{array}$ & 9 & NR \\
\hline & & $258 / 7,234$ & $49 / 2,138$ & $\begin{array}{l}\text { RR } 1.9 \text { (1.4-2.7) } \\
\text { (self-reported) }\end{array}$ & & & & 8 & \\
\hline $\begin{array}{l}\text { Englert et al. } \\
\text { (2001) (10) }\end{array}$ & $\begin{array}{c}100 \% \\
100 \% \mathrm{C}\end{array}$ & $2 / 458$ & $1 / 687$ & $\begin{array}{c}\text { RR } 3.01 \\
(0.27-33.28)\end{array}$ & 15 & $\begin{array}{l}\text { Age, sex, clinic, } \\
\text { calendar year }\end{array}$ & $\begin{array}{c}16 \text { plastic surgery } \\
\text { practices }\end{array}$ & 9 & Yes \\
\hline $\begin{array}{l}\text { Edworthy } \\
\text { et al. (1998) } \\
\text { (11) }\end{array}$ & $\begin{array}{c}71 \% ; \\
100 \% \text { C }\end{array}$ & $11 / 1,576$ & $6 / 725$ & $\begin{array}{c}\text { RR } 1.44 \\
(0.50-4.15)\end{array}$ & 12 & Age, sex & $\begin{array}{c}\text { Canadian } \\
\text { provincial health } \\
\text { registry }\end{array}$ & 8 & No \\
\hline
\end{tabular}

Cohort studies comparing with breast reduction surgeries

$\begin{array}{lcccccc}\text { Nyren et al. } & 56 \% ; & 11 / 7,442 & 5 / 3,353 & \text { RR } 1.3(0.7-2.5) & 8.0 & \text { Age, sex, follow-up Swedish national } 8 \quad \text { Yes } \\ \text { (1998) (13) } & 100 \% \text { C } & & & & \end{array}$

Cohort studies comparing with women from the community without breast implants

\begin{tabular}{|c|c|c|c|c|c|c|c|c|c|}
\hline \multirow[t]{2}{*}{$\begin{array}{l}\text { Watad et al. } \\
(2018)(14)\end{array}$} & $\begin{array}{l}100 \% \\
95 \% \mathrm{C}\end{array}$ & $278 / 24,651$ & $970 / 98,604$ & $\begin{array}{c}\text { OR } 1.19 \text { (1.03-1.38) } \\
\text { (prevalence) }\end{array}$ & \multirow[t]{2}{*}{9.7} & \multirow{2}{*}{$\begin{array}{l}\text { Age, sex, SES, } \\
\text { smoking, breast } \\
\text { cancer }\end{array}$} & \multirow[t]{2}{*}{$\begin{array}{c}\text { Israeli healthcare } \\
\text { database }\end{array}$} & 8 & No \\
\hline & & $7 / 1,797$ & $17 / 7,109$ & $\begin{array}{c}\text { HR } 1.75 \begin{array}{c}(0.70-4.33) \\
\text { (incidence) }^{*}\end{array}\end{array}$ & & & & 9 & \\
\hline $\begin{array}{l}\text { Gabriel et al. } \\
(1994)(15)\end{array}$ & $\begin{array}{l}85 \% \\
71 \% \text { C }\end{array}$ & $0 / 749$ & $2 / 1,498$ & RR 0 & 7.8 & N/A & $\begin{array}{l}\text { Tertiary care and } \\
\text { affiliated centers }\end{array}$ & 8 & No \\
\hline \multicolumn{10}{|c|}{ Cohort studies comparing with female health professionals without breast implants } \\
\hline $\begin{array}{l}\text { Sanchez- } \\
\text { Guerrero et al. } \\
\text { (1995) (16) }\end{array}$ & $\begin{array}{l}74 \% \\
50 \% \text { C }\end{array}$ & $3 / 1,183$ & $389 / 86,318$ & RR $0.9(0.3-2.6)$ & 9.9 & Age, sex & $\begin{array}{l}\text { Nurses' Health } \\
\text { Study }\end{array}$ & 7 & Yes \\
\hline $\begin{array}{l}\text { Hennekens } \\
\text { et al. (1996) } \\
\text { (17) }\end{array}$ & $\begin{array}{l}\text { NR; \% C } \\
\text { NR }\end{array}$ & 107/10,830 & $6,322 / 384,713$ & $\begin{array}{c}\text { HR } 1.18 \\
(0.97-1.43) \\
\text { (self-reported) }\end{array}$ & $<4-\geq 10$ & $\begin{array}{l}\text { Age, sex, calendar } \\
\text { year, cancer, implant } \\
\text { duration }\end{array}$ & $\begin{array}{c}\text { Women's Health } \\
\text { Study }\end{array}$ & 6 & Yes \\
\hline $\begin{array}{l}\text { Lee et al. } \\
\text { (2011) (18) }\end{array}$ & $\begin{array}{l}70 \% \\
68 \% \mathrm{C}\end{array}$ & $12 / 3,950$ & $32 / 19,897$ & $\begin{array}{c}\text { HR } 1.30 \\
(0.56-3.04)\end{array}$ & 3.6 & $\begin{array}{l}\text { Age, sex, body } \\
\text { mass index, } \\
\text { smoking, hormone, } \\
\text { cancer }\end{array}$ & $\begin{array}{c}\text { Women's Health } \\
\text { Study }\end{array}$ & 5 & Yes \\
\hline \multicolumn{10}{|c|}{ Cohort study comparing with post-menopausal women } \\
\hline $\begin{array}{l}\text { Rubin et al. } \\
\text { (2010) (37) }\end{array}$ & $\begin{array}{c}\text { 67\%; } \\
100 \% \text { C }\end{array}$ & $67 / 1,241$ & $4,545 / 85,350$ & $\begin{array}{c}P=0.367 \\
\text { (self-reported) }\end{array}$ & & $\begin{array}{l}\text { Age, sex; breast } \\
\text { cancer excluded }\end{array}$ & $\begin{array}{c}\text { Women's Health } \\
\text { Initiative; } \\
\text { prevalent? }\end{array}$ & 5 & No \\
\hline
\end{tabular}

Table 3 (continued) 
Table 3 (continued)

\begin{tabular}{|c|c|c|c|c|c|c|c|c|c|}
\hline $\begin{array}{l}\text { Study (year) } \\
\text { (reference) }\end{array}$ & $\begin{array}{c}\% \text { in } \\
\text { silicone; } \\
\% \text { C }\end{array}$ & $\begin{array}{c}\text { Implant } \\
\text { group, } \mathrm{n} / \mathrm{N}^{*}\end{array}$ & $\begin{array}{l}\text { Comparator } \\
\text { group, } \mathrm{n} / \mathrm{N}^{\star}\end{array}$ & $\begin{array}{l}\text { Measure of effect } \\
\qquad(95 \% \mathrm{Cl})\end{array}$ & $\begin{array}{c}\text { Follow-up, } \\
\text { years }\end{array}$ & Adjustments & Notes & NOS & $\$$ \\
\hline $\begin{array}{l}\text { Park et al. } \\
\text { (1998) (30) }\end{array}$ & $\begin{array}{l}100 \% \\
0 \% \text { C }\end{array}$ & $1 / 207$ & $1 / 88$ & $\begin{array}{c}\text { OR } 0.62 \\
(0.02-23.05)\end{array}$ & 5.9 & $\begin{array}{l}\text { Age, sex, cancer } \\
\text { stage, time of } \\
\text { surgery }\end{array}$ & $\begin{array}{c}\text { Inpatient unit; } \\
\text { unclear if prevalent } \\
\text { diagnoses }\end{array}$ & 6 & No \\
\hline
\end{tabular}

Cohort studies comparing with national rates

\begin{tabular}{|c|c|c|c|c|c|c|c|c|c|}
\hline $\begin{array}{l}\text { Fryzek et al. } \\
(2007)(8)\end{array}$ & $\begin{array}{l}>84 \% \\
100 \% \text { C }\end{array}$ & $\begin{array}{c}17 / 2,761 \\
(15 \text { confirmed })\end{array}$ & NR & SIR 1.3 (0.8-2.2) & 13.4 & $\begin{array}{c}\text { Age, sex, calendar } \\
\text { period }\end{array}$ & $\begin{array}{c}\text { Danish national } \\
\text { hospital registry + } \\
\text { private clinics }\end{array}$ & 9 & NR \\
\hline \multirow[t]{2}{*}{$\begin{array}{l}\text { Coroneos } \\
\text { et al. (2019) } \\
\text { (2) }\end{array}$} & $\begin{array}{l}100 \% \\
83 \% \mathrm{C}\end{array}$ & $\begin{array}{l}349 / 41,975 \\
\text { MemoryGel }\end{array}$ & $\begin{array}{l}5.4 / 10,000 \\
\text { person-y }\end{array}$ & $\begin{array}{c}\text { SIR 1.11 } \\
(0.86-1.41) \\
\text { (self-reported) }\end{array}$ & 7 & Age, sex, race & $\begin{array}{l}\text { United States LPAS } \\
21 \% 3-y \text { follow-up }\end{array}$ & 7 & No \\
\hline & $\begin{array}{l}100 \% \\
87 \% \text { C }\end{array}$ & $\begin{array}{c}4 / 41,342 \\
\text { Natrelle }\end{array}$ & & $\begin{array}{c}\text { SIR } 0.15 \\
(0.04-0.38)\end{array}$ & 2 & Age, sex, race & $\begin{array}{l}\text { United States LPAS } \\
61 \% 2-y \text { follow-up }\end{array}$ & 7 & \\
\hline $\begin{array}{l}\text { Nyren et al. } \\
\text { (1998) (13) }\end{array}$ & $\begin{array}{l}56 \% ; \\
87 \% \text { C }\end{array}$ & $\begin{array}{c}\text { 19/7,442 } \\
\text { (8 prevalent, } 1 \\
\text { misclassified) }\end{array}$ & NR & $\begin{array}{l}\text { SHR } 1.0 \text { (0.6-1.5) } \\
\text { (includes prevalent } \\
\text { and misclassified) }\end{array}$ & 8.0 & $\begin{array}{c}\text { Age, sex, calendar } \\
\text { year }\end{array}$ & $\begin{array}{l}\text { Swedish national } \\
\text { inpatient registry }\end{array}$ & 7 & Yes \\
\hline \multicolumn{10}{|c|}{ Case-control studies } \\
\hline $\begin{array}{l}\text { Goldman } \\
\text { et al. (1995) } \\
\text { (23) }\end{array}$ & $\begin{array}{c}85 \% ; \% \\
\text { C NR }\end{array}$ & $\begin{array}{c}9 / 392 \\
\text { (4 prevalent) }\end{array}$ & $138 / 3,508$ & $\begin{array}{c}\text { OR } 0.84 \\
(0.41-1.62)\end{array}$ & 8.3 (cases) & $\begin{array}{l}\text { Age, sex, income, } \\
\text { period }\end{array}$ & $\begin{array}{c}\text { Rheumatology } \\
\text { practice controls, } \\
\text { no CTD or RA }\end{array}$ & 6 & Yes \\
\hline $\begin{array}{l}\text { Wolfe et al. } \\
\text { (1999) (38) }\end{array}$ & $\begin{array}{l}100 \% ; \\
\% \text { C NR }\end{array}$ & $3 / 464$ & $\begin{array}{c}\text { (I) } 2 / 261 \text {; (II) } \\
1 / 503\end{array}$ & $\begin{array}{c}\text { (I) OR } 0.84 \text { (0.14- } \\
5.05),(\text { II) OR } 3.28 \\
(0.34-31.66)\end{array}$ & NR & $\begin{array}{l}\text { Sex; no difference } \\
\text { if adjusted for age }\end{array}$ & $\begin{array}{l}\text { (I) Osteoarthritis; (II) } \\
\text { community }\end{array}$ & 4 & Yes \\
\hline
\end{tabular}

*, for case-control studies, case group (n breast implants/N); control group (n breast implants/N). In Watad et al., disease-specific HRs were obtained by personal communication. C, cosmetic augmentation; Cl, confidence interval; CTD, connective tissue disease; HR, hazard ratio; N/A, not applicable; NOS, Newcastle-Ottawa scale; NR, not reported; OR, odds ratio; RA, rheumatoid arthritis; RR, relative risk; SES, socioeconomic status; SHR, standardized hospitalization ratio; SIR, standardized incidence ratio; y, years; $\$$, potential financial or other conflict of interest.

pleuritis $(15,29,31)$, proteinuria or kidney disease $(31)$, cytopenia (31), or livedo reticularis (10), although CIs were often wide.

\section{Risk of fibromyalgia and/or CFS}

Four high-quality cohort studies examined the risk of incident diagnoses of fibromyalgia and/or CFS with sufficient follow-up time (Table 5) (42-44). In the Israeli database by Watad et al. (14), after excluding patients with any prevalent autoimmune or rheumatic disease, the HR was 2.24 (95\% CI: 1.47-3.39) for being diagnosed with fibromyalgia and/or CFS among 1,797 women with breast implants compared to 7,109 women from the community (personal communication).

In the study by Brinton et al., the risk of self-reported CFS was higher among 7,234 women with cosmetic breast implants compared to those with other cosmetic surgeries (RR 2.4, 95\% CI: 1.6-3.6) (9). When stratified according to the period of diagnosis, the risk estimates were higher during or after 1992, year during which the risks of breast implants were highly mediatized: for CFS, the RR increased 
Table 4 Summary of studies reporting on the association between breast implants and risk of systemic lupus erythematosus

\begin{tabular}{|c|c|c|c|c|c|c|c|c|c|}
\hline $\begin{array}{l}\text { Study (year) } \\
\text { (reference) }\end{array}$ & $\begin{array}{c}\% \text { in } \\
\text { silicone; } \\
\text { \% C }\end{array}$ & $\begin{array}{l}\text { Implant group, } \\
\qquad \mathrm{n} / \mathrm{N}^{\star}\end{array}$ & $\begin{array}{l}\text { Comparator } \\
\text { group, } \mathrm{n} / \mathrm{N}^{*}\end{array}$ & $\begin{array}{l}\text { Measure of effect } \\
\qquad(95 \% \mathrm{Cl})\end{array}$ & $\begin{array}{c}\text { Follow-up, } \\
\text { years }\end{array}$ & Adjustments & Notes & NOS & $\$$ \\
\hline $\begin{array}{l}\text { Fryzek et al. } \\
(2007)(8)\end{array}$ & $\begin{array}{l}>84 \% \\
100 \% \text { C }\end{array}$ & $0 / 2,761$ & $11 / 8,807$ & HR NR & 13.4 & $\begin{array}{c}\text { Age, sex, } \\
\text { calendar year, } \\
\text { clinic, time since } \\
\text { operation }\end{array}$ & $\begin{array}{c}\text { Danish national } \\
\text { hospital registry + } \\
\text { private clinics }\end{array}$ & 9 & NR \\
\hline
\end{tabular}

Cohort studies comparing with other cosmetic surgeries

\begin{tabular}{|c|c|c|c|c|c|c|c|c|}
\hline $\begin{array}{l}\text { Englert et al. } \\
(2001)(10)\end{array}$ & $\begin{array}{c}100 \% ; \\
100 \% \text { C }\end{array}$ & $0 / 458$ & $3 / 687$ & RR 0 & 15 & $\begin{array}{l}\text { Age, sex, clinic, } \\
\text { calendar year; } \\
\text { excluded cancer }\end{array}$ & $\begin{array}{l}16 \text { plastic surgery } \\
\text { practices }\end{array}$ & 9 \\
\hline $\begin{array}{l}\text { Brinton et al. } \\
(2004)(9)\end{array}$ & $\begin{array}{c}50 \% ; \\
100 \% \text { C }\end{array}$ & $72 / 7,234$ & $10 / 2,138$ & $\begin{array}{c}\text { RR } 2.1 \text { (1.1-4.2) } \\
\text { (self-reported) }\end{array}$ & 12.1 & $\begin{array}{l}\text { Age, sex, race, } \\
\text { calendar period }\end{array}$ & $\begin{array}{c}18 \text { plastic surgery } \\
\text { practices }\end{array}$ & 8 \\
\hline $\begin{array}{l}\text { Edworthy et al. } \\
\text { (1998) (11) }\end{array}$ & $\begin{array}{c}71 \% \\
100 \% \mathrm{C}\end{array}$ & $3 / 1,576$ & $3 / 725$ & RR 0.94 (0.17-5.23) & 12 & Age, sex & $\begin{array}{l}\text { Canadian provincial } \\
\text { health registry }\end{array}$ & 8 \\
\hline $\begin{array}{l}\text { Wells et al. } \\
\text { (1994) (12) }\end{array}$ & $\begin{array}{l}100 \% \\
\% \text { C NR }\end{array}$ & $0 / 222$ & $0 / 80$ & RR not calculable & $<5$ & N/A & $\begin{array}{c}\text { Single plastic } \\
\text { surgery practice }\end{array}$ & 6 \\
\hline
\end{tabular}

Cohort studies comparing with breast reduction surgeries

$\begin{array}{lccccccc}\text { Nyren et al. } & 56 \% ; & 3 / 7,442 & 3 / 3353 & \text { RR } 0.7(0.3-1.6) & 8.0 & \begin{array}{l}\text { Age, sex, } \\ \text { follow-up }\end{array} \text { inpatient registry } & \end{array}$

Cohort studies comparing with women from the community without breast implants

\begin{tabular}{|c|c|c|c|c|c|}
\hline \multirow[t]{2}{*}{$\begin{array}{l}\text { Watad et al. } \\
(2018)(14)\end{array}$} & $\begin{array}{l}100 \% \\
95 \% \text { C }\end{array}$ & $117 / 24,651$ & $457 / 98,604$ & $\begin{array}{l}\text { OR } 1.05 \text { (0.84-1.30) } \\
\quad \text { (prevalence) }\end{array}$ & 9.7 \\
\hline & & $2 / 1,797$ & $7 / 7,109$ & $\begin{array}{l}\text { HR } 0.78(0.11-5.6) \\
\text { (incidence) }{ }^{*}\end{array}$ & \\
\hline $\begin{array}{l}\text { Gabriel et al. } \\
\text { (1994) (15) }\end{array}$ & $\begin{array}{l}85 \% \\
71 \% \text { C }\end{array}$ & $0 / 749$ & $0 / 1,498$ & RR not calculable & 7.8 \\
\hline
\end{tabular}

Cohort studies comparing with female health professionals without breast implants

\begin{tabular}{|c|c|c|c|c|c|}
\hline $\begin{array}{l}\text { Sanchez- } \\
\text { Guerrero et al. } \\
\text { (1995) (16) }\end{array}$ & $\begin{array}{c}74 \% \\
50 \% \text { C }\end{array}$ & $0 / 1,183$ & $96 / 86,318$ & RR 0 & 9.9 \\
\hline $\begin{array}{l}\text { Hennekens } \\
\text { et al. (1996) (17) }\end{array}$ & $\begin{array}{c}\text { NR; } \\
100 \% \mathrm{C}\end{array}$ & $32 / 10,830$ & $1,561 / 384,713$ & $\begin{array}{c}\text { HR } 1.15 \text { (0.81-1.63) } \\
\text { (self-reported) }\end{array}$ & $<4-\geq 10$ \\
\hline $\begin{array}{l}\text { Lee et al. (2011) } \\
\text { (18) }\end{array}$ & $\begin{array}{l}70 \% \\
68 \% \mathrm{C}\end{array}$ & $8 / 3,950$ & $19 / 19,897$ & $\begin{array}{l}\text { HR } 2.27 \text { (0.93-5.54) } \\
\quad \text { (self-reported) }\end{array}$ & 3.6 \\
\hline
\end{tabular}

Cohort study comparing with post-mastectomy reconstructive surgery without implants
Age, sex, SES, Israeli healthcare 8 No smoking, breast database cancer

N/A

N/A

Age, sex, calendar year, cancer, implant duration
Age, sex, body Women's Health 5 Yes mass index, Study smoking, hormone, cancer
Greenland et al. NR;
$17 / 31,820$
NR
RR 2.74 (1.66-4.55) Limited
(2000) (19) \% C NR person-y

Table 4 (continued) 
Table 4 (continued)

\begin{tabular}{|c|c|c|c|c|c|c|c|c|c|}
\hline $\begin{array}{l}\text { Study (year) } \\
\text { (reference) }\end{array}$ & $\begin{array}{l}\% \text { in } \\
\text { silicone; } \\
\% \text { C }\end{array}$ & $\begin{array}{l}\text { Implant group, } \\
\qquad \mathrm{n} / \mathrm{N}^{*}\end{array}$ & $\begin{array}{l}\text { Comparator } \\
\text { group, } \mathrm{n} / \mathrm{N}^{\star}\end{array}$ & $\begin{array}{l}\text { Measure of effect } \\
\quad(95 \% \mathrm{Cl})\end{array}$ & $\begin{array}{l}\text { Follow-up, } \\
\text { years }\end{array}$ & Adjustments & Notes & NOS & $\$$ \\
\hline $\begin{array}{l}\text { Fryzek et al. } \\
(2007)(8)\end{array}$ & $\begin{array}{l}>84 \% \\
100 \% \text { C }\end{array}$ & $\begin{array}{l}2 / 2,761(0 \\
\text { confirmed) }\end{array}$ & NR & SIR $0.9(0.1-2.9)$ & 13.4 & $\begin{array}{c}\text { Age, sex, } \\
\text { calendar period }\end{array}$ & $\begin{array}{c}\text { Danish national } \\
\text { hospital registry + } \\
\text { private clinics }\end{array}$ & 9 & NR \\
\hline $\begin{array}{l}\text { Coroneos et al. } \\
\text { (2019) (2) }\end{array}$ & $\begin{array}{l}100 \% \\
87 \% \text { C }\end{array}$ & $\begin{array}{l}3 / 41,342 \\
\text { (Natrelle) }\end{array}$ & & SIR $0.11(0.02-0.32)$ & 2 & Age, sex, race & $\begin{array}{l}\text { United States LPAS } \\
61 \% 2-y \text { follow-up }\end{array}$ & 7 & No \\
\hline $\begin{array}{l}\text { Singh et al. } \\
(2017)(3)\end{array}$ & $\begin{array}{l}100 \% \\
87 \% \text { C }\end{array}$ & $12 / 40,396$ & $\begin{array}{c}54.4 / 100,000 \\
\text { person-y }\end{array}$ & $\begin{array}{c}\text { SIR } 0.1 \\
\text { (95\% CI: NR) } \\
\text { (lupus/lupus-like) }\end{array}$ & $5-8$ & Age, sex, race & $\begin{array}{l}\text { United States LPAS } \\
61 \% 5 \text {-y follow-up }\end{array}$ & 7 & Yes \\
\hline \multicolumn{10}{|c|}{ Case-control studies } \\
\hline $\begin{array}{l}\text { Goldman et al. } \\
\text { (1995) (23) }\end{array}$ & $\begin{array}{l}85 \% ; \% \\
\text { C NR }\end{array}$ & $\begin{array}{c}1 / 179 \\
\text { (prevalent) }\end{array}$ & $138 / 3,508$ & OR 0.14 (0.02-1.23) & $\begin{array}{c}8.3 \\
\text { (cases) }\end{array}$ & $\begin{array}{c}\text { Age, sex, } \\
\text { income, period }\end{array}$ & $\begin{array}{l}\text { Rheumatology } \\
\text { practice controls, no } \\
\text { CTD or RA }\end{array}$ & 6 & Yes \\
\hline $\begin{array}{l}\text { Bengtsson et al. } \\
\text { (2002) (39) }\end{array}$ & $\begin{array}{c}100 \% ; \% \\
\text { C NR }\end{array}$ & $3 / 85$ & $1 / 205$ & OR NR & NR & N/A & Community controls & 6 & No \\
\hline $\begin{array}{l}\text { Strom et al. } \\
\text { (1994) (40) }\end{array}$ & $\begin{array}{c}100 \% ; \% \\
\text { C NR }\end{array}$ & $1 / 133$ & $8 / 4,754$ & $\begin{array}{c}\text { OR } 4.5 \\
\text { (90\% Cl: } 0.2-27.3)\end{array}$ & 8 (cases) & Sex & Community controls & 3 & No \\
\hline
\end{tabular}

*, for case-control studies, case group (n breast implants/N); control group (n breast implants/N). In Watad et al., disease-specific HRs were obtained by personal communication. C, cosmetic augmentation; Cl, confidence interval; CTD, connective tissue disease; HR, hazard ratio; N/A, not applicable; NOS, Newcastle-Ottawa scale; NR, not reported; OR, odds ratio; RA, rheumatoid arthritis; RR, relative risk; SES, socioeconomic status; SHR, standardized hospitalization ratio; SIR, standardized incidence ratio; y, years; \$, potential financial or other conflict of interest.

from 1.9 (95\% CI: $1.1-3.2)$ to 3.3 (95\% CI: $1.7-6.3)$, and for fibromyalgia, the RR increased from 0.9 (95\% CI: 0.61.4) to 1.9 (95\% CI: $1.2-3.0)$.

In the Danish cohort, the risk of fibromyalgia was not higher among 2,761 women with cosmetic breast implants compared to those with other cosmetic surgeries (HR 1.2, 95\% CI: 0.6-2.1) (8). However, compared to national rates standardized for age, sex and calendar period, both women with breast implants (SIR 1.9, 95\% CI: 1.6-2.2) and with other cosmetic surgeries (SIR 1.5, 95\% CI: 1.4-1.7) had an increased incidence of unspecified rheumatism, including fibromyalgia and myalgia.

Finally, Nyren et al. reported no increased risk of fibromyalgia among 7,442 women with breast implants identified in the Swedish national inpatient registry, compared to women with breast reductions (RR 1.0, 95\% CI: 0.3-3.0) (13). However, this study was limited to hospitalization data and would have missed fibromyalgia cases managed in outpatient settings.

Several studies also explored the risk of symptoms associated with fibromyalgia. Englert et al. found that among 458 women with breast implants identified from 16 plastic surgery practices, clusters of symptoms including myalgia, stiffness, low energy, pins and needles and poor memory, among others, were increased over two-fold compared to women with non-silicone plastic surgeries (27). Fryzek et al. also found that women with breast implants from an inpatient Swedish registry had a 1.3- to 1.5 -fold 
Table 5 Summary of studies reporting on the association between breast implants and risk of fibromyalgia and/or chronic fatigue syndrome

\begin{tabular}{|c|c|c|c|c|c|c|c|c|c|}
\hline $\begin{array}{l}\text { Study (year) } \\
\text { (reference) }\end{array}$ & $\begin{array}{c}\% \text { in } \\
\text { silicone; } \\
\% \text { C }\end{array}$ & $\begin{array}{c}\text { Implant group, } \\
\mathrm{n} / \mathrm{N}^{\star}\end{array}$ & $\begin{array}{l}\text { Comparator } \\
\text { group, } \mathrm{n} / \mathrm{N}^{*}\end{array}$ & $\begin{array}{l}\text { Measure of effect } \\
\qquad(95 \% \mathrm{Cl})\end{array}$ & $\begin{array}{c}\text { Follow-up, } \\
\text { years }\end{array}$ & Adjustments & Notes & NOS & $\$$ \\
\hline
\end{tabular}

Cohort studies comparing with other cosmetic surgeries and breast reduction surgeries

$\begin{array}{lcccc}\text { Fryzek et al. } & >84 \% ; & 17 / 2,761 & 37 / 8,807 & \text { HR } 1.2(0.6-2.1) \\ \text { (2007) (8) } & 100 \% \text { C } & \text { (FM) }\end{array}$

Cohort studies comparing with other cosmetic surgeries

\begin{tabular}{|c|c|c|c|c|}
\hline \multirow[t]{2}{*}{$\begin{array}{l}\text { Brinton et al. } \\
(2004)(9)\end{array}$} & $\begin{array}{c}50 \% \\
100 \% \mathrm{C}\end{array}$ & $311 / 7,234$ & $57 / 2,138$ & $\begin{array}{c}\text { RR } 1.3 \text { (0.9-1.7) } \\
\text { (FM, self-reported) }\end{array}$ \\
\hline & & $246 / 7,234$ & $27 / 2,138$ & $\begin{array}{c}\text { RR } 2.4 \text { (1.6-3.6) } \\
\text { (CFS, self-reported) }\end{array}$ \\
\hline $\begin{array}{l}\text { Englert et al. } \\
\text { (2001) (10) }\end{array}$ & $\begin{array}{c}100 \% \\
100 \% \mathrm{C}\end{array}$ & $1 / 329$ & $3 / 377$ & $\begin{array}{c}\text { OR } 0.38 \text { (0.04-3.67) } \\
\text { (FM, prevalent) }\end{array}$ \\
\hline
\end{tabular}

\section{Cohort studies comparing with breast reduction surgeries}

\begin{tabular}{|c|c|c|c|c|c|c|c|c|}
\hline $\begin{array}{l}\text { Nyren et al. } \\
\text { (1998) (13) }\end{array}$ & $\begin{array}{c}56 \% ; \\
100 \% \text { C }\end{array}$ & $8 / 7,442$ & $4 / 3,353$ & $\begin{array}{c}\text { RR } 1.0(0.3-3.0) \\
(F M)\end{array}$ & 8.0 & $\begin{array}{l}\text { Age, sex, } \\
\text { follow-up }\end{array}$ & $\begin{array}{l}\text { Swedish national } \\
\text { inpatient registry }\end{array}$ & 8 \\
\hline $\begin{array}{l}\text { Breiting et al. } \\
(2004)(33)\end{array}$ & $\begin{array}{c}100 \% ; \\
100 \% \text { C }\end{array}$ & $13 / 190$ & $17 / 186$ & $\begin{array}{c}\text { OR } 0.7(0.3-1.5) \\
\text { (FM/post-infectious } \\
\text { arthritis, } \\
\text { self-reported) }\end{array}$ & 19 & $\begin{array}{c}\text { Age, sex, } \\
\text { date of surgery, } \\
\text { hospital/clinic }\end{array}$ & $\begin{array}{l}1 \text { public hospital, } \\
1 \text { private plastic } \\
\text { surgery practice }\end{array}$ & 5 \\
\hline
\end{tabular}

Cohort studies comparing with women from the community without breast implants

\begin{tabular}{|c|c|c|c|c|c|c|c|c|c|}
\hline \multirow[t]{2}{*}{$\begin{array}{l}\text { Watad et al. } \\
(2018)(14)\end{array}$} & $\begin{array}{l}100 \% \\
95 \% \text { C }\end{array}$ & $1,997 / 24,651$ & $6,106 / 98,604$ & $\begin{array}{c}\text { OR } 1.37 \\
(1.29-1.45)(\mathrm{FM} / \\
\text { CFS, prevalence) }\end{array}$ & 9.7 & $\begin{array}{l}\text { Age, sex, SES, } \\
\text { smoking, breast } \\
\text { cancer }\end{array}$ & $\begin{array}{c}\text { Israeli healthcare } \\
\text { database }\end{array}$ & 8 & No \\
\hline & & $38 / 1,797$ & $72 / 7,109$ & $\begin{array}{c}\text { HR } 2.24 \text { (1.47-3.39) } \\
\text { (FM/CFS, } \\
\text { incidence) }{ }^{\star}\end{array}$ & & & & 9 & \\
\hline $\begin{array}{l}\text { Breiting et al. } \\
\text { (2004) (33) }\end{array}$ & $\begin{array}{c}100 \% ; \\
100 \% \text { C }\end{array}$ & $13 / 190$ & $10 / 149$ & $\begin{array}{c}\text { OR } 1.0(0.4-2.4) \\
\text { (FM/post-infectious } \\
\text { arthritis, } \\
\text { self-reported) }\end{array}$ & 19 & Age, sex, date & $\begin{array}{l}1 \text { public hospital, } \\
1 \text { private plastic } \\
\text { surgery practice }\end{array}$ & 5 & Yes \\
\hline $\begin{array}{l}\text { Khoo et al. } \\
\text { (2019) (42) }\end{array}$ & $\begin{array}{l}100 \% \\
\% \text { C NR }\end{array}$ & $6 / 30$ & $\begin{array}{l}1 / 45 \text { SSc, } \\
8 / 45 \text { SLE }\end{array}$ & $\begin{array}{c}P=0.01(\mathrm{SSc}) \\
\mathrm{P}=1.00 \text { (SLE) } \\
(\mathrm{FM} / \mathrm{CFS})\end{array}$ & 16.1 & Age, sex & $\begin{array}{l}\text { Rheumatology } \\
\text { practice }\end{array}$ & 6 & No \\
\hline $\begin{array}{l}\text { Fryzek et al. } \\
(2007)(8)\end{array}$ & $\begin{array}{l}>84 \% \\
100 \% \text { C }\end{array}$ & $175 / 2,761$ & NR & $\begin{array}{l}\text { SIR } 1.9 \text { (1.6-2.2) } \\
\text { (unspecified } \\
\text { rheumatisms, } \\
\text { including FM) }\end{array}$ & 13.4 & $\begin{array}{l}\text { Age, sex, calendar } \\
\text { period }\end{array}$ & $\begin{array}{c}\text { Danish national } \\
\text { hospital registry + } \\
\text { private clinics }\end{array}$ & 9 & NR \\
\hline
\end{tabular}

Table 5 (continued) 
Table 5 (continued)

\begin{tabular}{|c|c|c|c|c|c|c|c|c|c|}
\hline $\begin{array}{l}\text { Study (year) } \\
\text { (reference) }\end{array}$ & $\begin{array}{c}\% \text { in } \\
\text { silicone; } \\
\text { \% C }\end{array}$ & $\begin{array}{l}\text { Implant group, } \\
\qquad \mathrm{n} / \mathrm{N}^{*}\end{array}$ & $\begin{array}{l}\text { Comparator } \\
\text { group, } \mathrm{n} / \mathrm{N}^{*}\end{array}$ & $\begin{array}{l}\text { Measure of effect } \\
\qquad(95 \% \mathrm{Cl})\end{array}$ & $\begin{array}{c}\text { Follow-up, } \\
\text { years }\end{array}$ & Adjustments & Notes & NOS & $\$$ \\
\hline $\begin{array}{l}\text { Coroneos } \\
\text { et al. (2019) } \\
\text { (2) }\end{array}$ & $\begin{array}{l}100 \% \\
87 \% \mathrm{C}\end{array}$ & $\begin{array}{l}9 / 41,342 \\
\text { (Natrelle) }\end{array}$ & $\begin{array}{c}112.8 / 10,000 \\
\text { person-y }\end{array}$ & $\begin{array}{c}\text { SIR } 0.02(0.01-0.03) \\
\text { (FM) }\end{array}$ & 2 & Age, sex, race & $\begin{array}{l}\text { United States LPAS } \\
61 \% 2-y \text { follow-up }\end{array}$ & 7 & No \\
\hline \multicolumn{10}{|c|}{ Case-control studies } \\
\hline $\begin{array}{l}\text { Lai et al. } \\
(2000)(43)\end{array}$ & $\begin{array}{c}96 \% ; \% \\
\text { C NR }\end{array}$ & $16 / 484$ & $61 / 1,532$ & $\begin{array}{c}\text { OR } 0.74(0.42-1.32) \\
\text { (FM) }\end{array}$ & NR & $\begin{array}{c}\text { Age, sex, income, } \\
\text { CTD or RA, } \\
\text { hypermobility }\end{array}$ & $\begin{array}{l}\text { Rheumatology } \\
\text { practice controls } \\
\text { without FM }\end{array}$ & 7 & Yes \\
\hline $\begin{array}{l}\text { Wolfe et al. } \\
\text { (1999) (38) }\end{array}$ & $\begin{array}{l}100 \% \\
\% \text { C NR }\end{array}$ & $3 / 508$ & $2 / 261 ; 1 / 503$ & $\begin{array}{c}\text { OR } 3.01 \\
(0.31-29.05)\end{array}$ & NR & $\begin{array}{l}\text { Sex; no difference } \\
\text { if adjusted for age }\end{array}$ & Community controls & 4 & Yes \\
\hline
\end{tabular}

*, for case-control studies, case group (n breast implants/N); control group (n breast implants/N). In Watad et al., disease-specific HRs were obtained by personal communication. C, cosmetic augmentation; Cl, confidence interval; CFS, chronic fatigue syndrome; FM, fibromyalgia; HR, hazard ratio; N/A, not applicable; NOS, Newcastle-Ottawa scale; NR, not reported; OA, osteoarthritis; OR, odds ratio; SES, socioeconomic status; SHR, standardized hospitalization ratio; SIR, standardized incidence ratio; y, years; $\$$, potential financial or other conflict of interest.

increase in risk of reporting any of 28 rheumatic, sicca, cognitive or other systemic symptoms, compared to women undergoing breast reduction surgery (28). Notably, none of these exploratory studies adjusted for multiple comparisons.

\section{Risk of combinations and other autoimmune/inflammatory rheumatic diseases}

Few studies examined the association between breast implants and autoimmune myositis, MCTD, UCTD, vasculitis, spondyloarthropathies and sarcoidosis (Supplementary Files 3 to 5: https://cdn.amegroups.cn/static/public/gs-21-266-35.pdf) (2,3,8-10,13-19,23,33,45-47). Overall, their observed event rates were not higher than expected in the general population. Five cohort studies found no increased risk for combinations of incident and confirmed autoimmune/ inflammatory rheumatic diseases, with relative risks from 0.6 to $2.0(8,9,13,15,16,18)$ (Supplementary File 4: https://cdn. amegroups.cn/static/public/gs-21-266-4.pdf). In contrast, one high-quality cohort study by Watad et al. found an increased incident risk of developing any autoimmune or rheumatic disorder compared to community controls (HR 1.45, 95\% CI: $1.21-1.73$ ), with a combined endpoint that additionally included fibromyalgia/CFS and non-rheumatic autoimmune diseases (hypothyroidism, hyperthyroidism, multiple sclerosis and psoriasis) (14).

\section{Predictors and immunological markers of rbeumatic disease among breast implant users}

Studies on the association between breast implant rupture and rheumatic disease were underpowered to detect risk differences for CTDs (Supplementary File 6: https://cdn.amegroups.cn/static/public/gs-21-266-6.pdf) $(28,34,35,42,48-53)$. One study found a 1.3 to 1.5 -fold increase in risk of myalgias and Raynaud's phenomenon among breast implant patients with local complications (capsulotomy, implant change or implant leakage) compared to those without complications (28). Extracapsular silicone leakage detected on magnetic resonance imaging (MRI) 
was associated with a 3-fold increased risk in Raynaud's and fibromyalgia in one study (49), but not in another (48). Rupture was not associated with an increased risk of sicca $(28,49)$. No studies specifically examined the association between acute/traumatic implant rupture and time to rheumatic disease onset. Although saline-filled implants have typically been portrayed as a safer alternative to silicone implants, little data was available in regard to the association between saline implants and rheumatic diseases $(3,18)$ (Supplementary File 7: https://cdn.amegroups.cn/ static/public/gs-21-266-7.pdf). Few studies examined the effect of implant indication (cosmetic versus reconstructive) $(3,13,15,17,18)$ and implant duration $(17,18)$ on risk of rheumatic disease (Supplementary File 7: https://cdn. amegroups.cn/static/public/gs-21-266-7.pdf). We did not identify any study that specifically assessed the outcome of patients with pre-existing rheumatic disease undergoing breast implant surgery. No immunological marker reproducibly differentiated breast implant users with and without rheumatic symptoms (Supplementary File 8: https://cdn.amegroups.cn/static/public/gs-21-266-8.pdf) (54-72).

\section{Course of rheumatic disease after explantation}

Studies reporting on the course of rheumatic disease after explantation are presented in Supplementary File 9: https://cdn.amegroups.cn/static/public/gs-21-266-9.pdf (73-85). For non-specific rheumatic symptoms (including arthralgias, myalgias, fatigue or sicca), improvement was observed in $32-100 \%$ of patients and generally occurred immediately or within the first few months postexplantation (73-78,80-83,85). However, improvement was often temporary $(73,77,79,80)$. As for CTDs, Wallace et al. reported their experience in $16 \mathrm{SLE}$ and $10 \mathrm{SSc}$ patients who had an explantation (84). Subjective improvement was common, but transient, in the majority of patients. Notably, one patient with SSc developed renal crisis requiring dialysis within two months of explantation. Case reports and series with less than 20 patients were excluded from this systematic review due to their high risk of selection bias, but have been reviewed elsewhere (86). Overall, in studies reporting improvement, it is unclear whether the benefit attributed to explantation was due to removal of the breast implants, to the use of concomitant disease-modifying treatments (such as glucocorticoids and immunosuppressive drugs), to the natural history of disease (such as skin softening in SSc) or to a placebo effect.

\section{Discussion}

We conducted a rigorous systematic literature review to characterize the association between breast implants and risk of incident rheumatic disease. Overall, two cohort studies suggested a two-fold increase in risk of SSc, but with much uncertainty around the estimates, and three casecontrol studies showed no increase in risk in SSc. The risk of confirmed SS was not increased, however symptoms of sicca were reported more frequently among breast implant users. A meta-analysis of heterogenous studies reported a small increase in risk of RA (36). Studies did not support an association with SLE. Diagnoses and symptoms of fibromyalgia and/or CFS, which have overlapping symptoms with the "breast implant illness", were reported about twice as frequently among breast implant users compared to women from the community.

Admittedly, the study of causality between exposure to breast implants and rheumatic disease outcomes poses multiple epidemiological challenges. Ideally, to state causality, one must be able to demonstrate that the exposure to breast implants preceded the onset of the rheumatic disease (temporality), that increased exposure is associated with increased effect (biological gradient) and that the removal of the exposure leads to a reduction in disease risk (reversibility) (87). However, given that the pathophysiology of CTDs is often characterized by multiple hits (i.e., result from an accumulation of genetic and environment factors which eventually lead to disease expression), there may be different possible latency periods between breast implant surgery and disease onset which may span years to decades, making it difficult to assess risk over shorter follow-up periods.

There may also be different thresholds of exposure that will lead to disease in different individuals, depending on individual susceptibilities. Chronic leakage of small amounts of implant contents may not be readily measurable using MRI, and studies looking at immunological assays have failed to identify a reliable measure that correlates with clinical symptoms. It would be interesting to study whether acute/traumatic implant ruptures, which may represent larger "doses" of exposure to implant contents, are associated with a shorter time to developing the outcomes. In regard to reversibility with explantation, this may not be observed in multiple hit diseases, in which an exposure contributes to disease expression, but is not the sole cause. In fact, many autoimmune diseases result from immunological cascades that are irreversible once 
triggered.

Another challenge relates to the rarity of the studied outcomes, particularly for CTDs. The incidence rates of SSc, SS, RA and SLE in females living in the United States are as low as $0.6,0.7,5.4$ and 5.4 per 10,000 personyears (2). Thus, studies need to follow a very large number of individuals over a sufficiently long period of time in order to be powered to detect a difference in disease risk. However, this is not easily achieved, as reflected in two large post-approval studies which intended to follow over 40,000 individuals with breast implants over 10 years, but which unfortunately only succeeded in following about $20 \%$ and $60 \%$ of patients at 3 and 5 years $(2,3)$. Studies with significant loss in follow-up are at risk for differential selection bias, for example, if patients who experience symptoms are more likely to continue medical follow-up compared to asymptomatic patients, thus potentially leading to an overestimation of disease risk.

To overcome the limited sample sizes in individual studies, meta-analyses can be done. However, we decided against performing a quantitative synthesis given that too few homogeneous and high-quality studies remained after taking into consideration the following factors:

(I) Cosmetic breast implant users are very different from cancer patients with reconstructive surgery, who can have paraneoplastic syndromes and adverse effects from cancer therapies acting as additional confounders, and should not be combined without adequate adjustment.

(II) Women with other cosmetic surgeries, with breast reduction surgeries, from the community and from health professional groups are very different populations and should not be considered as comparable comparator groups.

(III) Self-reported diagnoses have low confirmation rates compared to chart validation $(9,88)$ and should not be used to assess the risk of defined rheumatic diseases. Studies including cases with misclassified diagnoses should also not be combined with studies with validated diagnoses.

(IV) Estimates largely based on prevalent diagnoses should not be included when assessing the risk of incident rheumatic disease.

(V) Studies with significant loss in follow-up are at risk for differential selection bias and should not be included in summary assessments, as explained above.

(VI) Studies with short follow-up should not be included, as patients may not have had the time to develop the disease, potentially leading to an underestimation of the risk.

(VII) Studies using only inpatient data will fail to capture rheumatic outcomes that are largely diagnosed and followed in outpatient settings, leading to an underestimation of the risk.

Despite these limitations, key findings can be highlighted from this systematic review. First, based on a small number of high-quality and methodologically robust studies, an association between breast implants and a small increased risk of SSc and RA could not be excluded. In addition, symptoms of sicca, myalgia and fatigue were more commonly reported by breast implant users. Nevertheless, in absolute numbers, rheumatic outcomes were rare among breast implant users. More studies are required to identify factors that will predict the risk of developing disease and more data is required on the safety of saline breast implants. Finally, the uncertainty surrounding the safety of breast implants needs to be communicated to patients in order to allow them to make an informed decision when weighing the benefits and potential risks.

\section{Conclusions}

In this up-to-date systematic review of high-quality and methodologically robust studies on the association between breast implants and rheumatic diseases, rheumatic outcomes were rare among breast implant users. A small increase in risk of SSc and RA could not be excluded. In addition, symptoms of sicca, myalgia and fatigue were more commonly reported by breast implant users. Individuals considering the placement of breast implants should be informed of the uncertainty surrounding the risk of rheumatic disease associated with breast implants.

\section{Acknowledgments}

We would like to thank Caroline Sauvé, librarian at the CHUM, for her time and assistance in developing and executing the systematic review search strategy. We would like to thank Vered Rosenberg (Maccabi Healthcare Services) for providing results from additional analyses for the purpose of this systematic review.

Funding: $\mathrm{SH}$ is supported by the Centre hospitalier de l'Université de Montréal (CHUM) Research Center, the CHUM Division of Rheumatology and Université de Montréal's Department of Medicine. 


\section{Footnote}

Reporting Checklist: The authors have completed the PRISMA reporting checklist. Available at https://dx.doi. org/10.21037/gs-21-266

Conflicts of Interest: All authors have completed the ICMJE uniform disclosure form (available at https://dx.doi. org/10.21037/gs-21-266). The authors have no conflicts of interest to declare.

Ethical Statement: The authors are accountable for all aspects of the work in ensuring that questions related to the accuracy or integrity of any part of the work are appropriately investigated and resolved.

Open Access Statement: This is an Open Access article distributed in accordance with the Creative Commons Attribution-NonCommercial-NoDerivs 4.0 International License (CC BY-NC-ND 4.0), which permits the noncommercial replication and distribution of the article with the strict proviso that no changes or edits are made and the original work is properly cited (including links to both the formal publication through the relevant DOI and the license). See: https://creativecommons.org/licenses/by-nc-nd/4.0/.

\section{References}

1. de Jong D, Vasmel WL, de Boer JP, et al. Anaplastic largecell lymphoma in women with breast implants. JAMA 2008;300:2030-5.

2. Coroneos CJ, Selber JC, Offodile AC 2nd, et al. US FDA Breast Implant Postapproval Studies: Long-term Outcomes in 99,993 Patients. Ann Surg 2019;269:30-6.

3. Singh N, Picha GJ, Hardas B, et al. Five-Year Safety Data for More than 55,000 Subjects following Breast Implantation: Comparison of Rare Adverse Event Rates with Silicone Implants versus National Norms and Saline Implants. Plast Reconstr Surg 2017;140:666-79.

4. Breast Implants - Certain Labeling Recommendations to Improve Patient Communication: US Food and Drug Administration; Document issued on September 29, 2020. [Accessed October 19, 2020]. Available online: https:// www.fda.gov/media/131885/download

5. Caravantes-Cortes MI, Roldan-Valadez E, ZwojewskiMartinez RD, et al. Breast Prosthesis Syndrome: Pathophysiology and Management Algorithm. Aesthetic Plast Surg 2020;44:1423-37.
6. Fuzzard SK, Teixeira R, Zinn R. A Review of the Literature on the Management of Silicone Implant Incompatibility Syndrome. Aesthetic Plast Surg 2019;43:1145-9.

7. Wells G, Shea B, O'Connell D, et al. The NewcastleOttawa Scale (NOS) for assessing the quality of nonrandomized studies in meta-analyses. Available online: http://www.ohri.ca/programs/clinical_epidemiology/ oxford.asp. 2009.

8. Fryzek JP, Holmich L, McLaughlin JK, et al. A nationwide study of connective tissue disease and other rheumatic conditions among Danish women with long-term cosmetic breast implantation. Ann Epidemiol 2007;17:374-9.

9. Brinton LA, Buckley LM, Dvorkina O, et al. Risk of connective tissue disorders among breast implant patients. Am J Epidemiol 2004;160:619-27.

10. Englert H, Joyner E, McGill N, et al. Women's health after plastic surgery. Intern Med J 2001;31:77-89.

11. Edworthy SM, Martin L, Barr SG, et al. A clinical study of the relationship between silicone breast implants and connective tissue disease. J Rheumatol 1998;25:254-60.

12. Wells KE, Cruse CW, Baker JL, Jr., et al. The health status of women following cosmetic surgery. Plast Reconstr Surg 1994;93:907-12.

13. Nyren O, Yin L, Josefsson S, et al. Risk of connective tissue disease and related disorders among women with breast implants: a nation-wide retrospective cohort study in Sweden. BMJ: British Medical Journal (International Edition) 1998;316:417-22.

14. Watad A, Rosenberg V, Tiosano S, et al. Silicone breast implants and the risk of autoimmune/rheumatic disorders: A real-world analysis. International Journal of Epidemiology 2018;47:1846-54.

15. Gabriel SE, O'Fallon WM, Kurland LT, et al. Risk of connective-tissue diseases and other disorders after breast implantation. N Engl J Med 1994;330:1697-702.

16. Sanchez-Guerrero J, Colditz GA, Karlson EW, et al. Silicone breast implants and the risk of connective-tissue diseases and symptoms. N Engl J Med 1995;332:1666-70.

17. Hennekens CH, Lee IM, Cook NR, et al. Self-reported breast implants and connective-tissue diseases in female health professionals. A retrospective cohort study. JAMA 1996;275:616-21.

18. Lee IM, Cook NR, Shadick NA, et al. Prospective cohort study of breast implants and the risk of connective-tissue diseases. Int J Epidemiol 2011;40:230-8.

19. Greenland S, Finkle WD. A retrospective cohort study of implanted medical devices and selected chronic diseases in Medicare claims data. Ann Epidemiol 2000;10:205-13. 
20. Englert H, Morris D, March L. Scleroderma and silicone gel breast prostheses--the Sydney study revisited. Aust N Z J Med 1996;26:349-55.

21. Burns CJ, Laing TJ, Gillespie BW, et al. The epidemiology of scleroderma among women: assessment of risk from exposure to silicone and silica. J Rheumatol 1996;23:1904-11.

22. Hochberg MC, Perlmutter DL, Medsger TA, Jr., et al. Lack of association between augmentation mammoplasty and systemic sclerosis (scleroderma). Arthritis Rheum 1996;39:1125-31.

23. Goldman JA, Greenblatt J, Joines R, et al. Breast implants, rheumatoid arthritis, and connective tissue diseases in a clinical practice. J Clin Epidemiol 1995;48:571-82.

24. Whorton D, Wong O. Scleroderma and silicone breast implants. West J Med 1997;167:159-65.

25. Saigusa R, Asano Y, Nakamura K, et al. Association of anti-RNA polymerase III antibody and silicone breast implants in patients with systemic sclerosis. J Dermatol 2016;43:808-10.

26. Lazzaroni MG, Campochiaro C, Bertoldo E, et al. Association of anti-RNA polymerase III antibody with silicone breast implants rupture in a multicentre series of Italian patients with systemic sclerosis. Clin Exp Rheumatol 2021;39 Suppl 131:25-8.

27. Englert H, Joyner E, Thompson M, et al. Augmentation mammoplasty and "silicone-osis". Intern Med J 2004;34:668-76.

28. Fryzek JP, Signorello LB, Hakelius L, et al. Local complications and subsequent symptom reporting among women with cosmetic breast implants. Plast Reconstr Surg 2001;107:214-21.

29. Kjoller K, Holmich LR, Fryzek JP, et al. Self-reported musculoskeletal symptoms among Danish women with cosmetic breast implants. Ann Plast Surg 2004;52:1-7.

30. Park AJ, Black RJ, Sarhadi NS, et al. Silicone gel-filled breast implants and connective tissue diseases. Plastic and Reconstructive Surgery 1998;101:261-8.

31. Giltay EJ, Bernelot Moens HJ, Riley AH, et al. Silicone breast prostheses and rheumatic symptoms: a retrospective follow up study. Ann Rheum Dis 1994;53:194-6.

32. Jensen B, Hechmann WI, Friis S, et al. Self-reported symptoms among Danish women following cosmetic breast implant surgery. Clin Rheumatol 2002;21:35-42.

33. Breiting VB, Holmich LR, Brandt B, et al. Long-term health status of Danish women with silicone breast implants. Plast Reconstr Surg 2004;114:217-26; discussion 27-8.

34. Berner I, Gaubitz M, Jackisch C, et al. Comparative examination of complaints of patients with breast-cancer with and without silicone implants. Eur J Obstet Gynecol Reprod Biol 2002;102:61-6.

35. Vermeulen RC, Scholte HR. Rupture of silicone gel breast implants and symptoms of pain and fatigue. J Rheumatol 2003;30:2263-7.

36. Balk EM, Earley A, Avendano EA, et al. Long-Term Health Outcomes in Women With Silicone Gel Breast Implants: A Systematic Review. Ann Intern Med 2016;164:164-75.

37. Rubin JP, Landfair AS, Shestak K, et al. Health characteristics of postmenopausal women with breast implants. Plast Reconstr Surg 2010;125:799-810.

38. Wolfe F, Anderson J. Silicone filled breast implants and the risk of fibromyalgia and rheumatoid arthritis. J Rheumatol 1999;26:2025-8.

39. Bengtsson AA, Rylander L, Hagmar L, et al. Risk factors for developing systemic lupus erythematosus: a casecontrol study in southern Sweden. Rheumatology (Oxford) 2002;41:563-71.

40. Strom BL, Reidenberg MM, Freundlich B, et al. Breast silicone implants and risk of systemic lupus erythematosus. J Clin Epidemiol 1994;47:1211-4.

41. Janowsky EC, Kupper LL, Hulka BS. Meta-analyses of the relation between silicone breast implants and the risk of connective-tissue diseases. N Engl J Med 2000;342:781-90.

42. Khoo T, Proudman S, Limaye V. Silicone breast implants and depression, fibromyalgia and chronic fatigue syndrome in a rheumatology clinic population. Clin Rheumatol 2019;38:1271-6.

43. Lai S, Goldman JA, Child AH, et al. Fibromyalgia, hypermobility, and breast implants. J Rheumatol 2000;27:2237-41.

44. MacDonald KL, Osterholm MT, LeDell KH, et al. A case-control study to assess possible triggers and cofactors in chronic fatigue syndrome. Am J Med 1996;100:548-54.

45. Barbosa MR, Makris UE, Mansi IA. Association of Breast Implants with Nonspecific Symptoms, Connective Tissue Diseases, and Allergic Reactions: A Retrospective Cohort Analysis. Plast Reconstr Surg 2021;147:42e-9e.

46. Laing TJ, Schottenfeld D, Lacey JV Jr, et al. Potential risk factors for undifferentiated connective tissue disease among women: implanted medical devices. Am J Epidemiol 2001;154:610-7.

47. Williams HJ, Weisman MH, Berry CC. Breast implants in patients with differentiated and undifferentiated connective tissue disease. Arthritis Rheum 1997;40:437-40.

48. Hölmich LR, Kjøller K, Fryzek JP, et al. Self-reported diseases and symptoms by rupture status among unselected 
Danish women with cosmetic silicone breast implants. Plast Reconstr Surg 2003;111:723-32; discussion 733-4.

49. Brown SL, Pennello G, Berg WA, et al. Silicone gel breast implant rupture, extracapsular silicone, and health status in a population of women. J Rheumatol 2001;28:996-1003.

50. Gaubitz M, Jackisch C, Domschke W, et al. Silicone breast implants: correlation between implant ruptures, magnetic resonance spectroscopically estimated silicone presence in the liver, antibody status and clinical symptoms. Rheumatology (Oxford) 2002;41:129-35; discussion 123-4.

51. Hölmich LR, Vejborg IM, Conrad C, et al. Untreated silicone breast implant rupture. Plast Reconstr Surg 2004;114:204-14; discussion 215-6.

52. Contant CM, Swaak AJ, Obdeijn AI, et al. A prospective study on silicone breast implants and the silicone-related symptom complex. Clin Rheumatol 2002;21:215-9.

53. Spendel S, Hintringer T, Pilz SM, et al. Clinical and laboratory investigation of women with intact and women with ruptured silicone gel breast implants. Eur J Plastic Surg 1998;21:338-43.

54. Claman HN, Robertson AD. Antinuclear antibodies and breast implants. West J Med 1994;160:225-8.

55. Bridges AJ, Conley C, Wang G, et al. A clinical and immunologic evaluation of women with silicone breast implants and symptoms of rheumatic disease. Ann Intern Med 1993;118:929-36.

56. Zandman-Goddard G, Blank M, Ehrenfeld M, et al. A comparison of autoantibody production in asymptomatic and symptomatic women with silicone breast implants. J Rheumatol 1999;26:73-7.

57. De Jong WH, Kallewaard M, Goldhoorn CA, et al. Longterm exposure to silicone breast implants does not induce antipolymer antibodies. Biomaterials 2004;25:1095-103.

58. Press RI, Peebles CL, Kumagai Y, et al. Antinuclear autoantibodies in women with silicone breast implants. Lancet 1992;340:1304-7.

59. Tenenbaum SA, Rice JC, Espinoza LR, et al. Use of antipolymer antibody assay in recipients of silicone breast implants. Lancet 1997;349:449-54.

60. Bridges AJ, Anderson JD, Burns DE, et al. Autoantibodies in patients with silicone implants. Curr Top Microbiol Immunol 1996;210:277-82.

61. Silverman S, Gluck O, Silver D, et al. The prevalence of autoantibodies in symptomatic and asymptomatic patients with breast implants and patients with fibromyalgia. Curr Top Microbiol Immunol 1996;210:317-22.

62. Karlson EW, Hankinson SE, Liang MH, et al. Association of silicone breast implants with immunologic abnormalities: a prospective study. Am J Med 1999;106:11-9.

63. Brunner CA, Feller AM, Gröner R, et al. Increase of immunologically relevant parameters in correlation with Baker classification in breast implant recipients. Ann Plast Surg 1996;36:512-18; discussion 518-21.

64. Contant CM, Swaak AJ, Wiggers T, et al. First evaluation study of the Dutch Working Party on silicone breast implants (SBI) and the silicone-related symptom complex (SRSC). Clin Rheumatol 2000;19:458-63.

65. Jensen B, Wiik A, Hechmann Wittrup I, et al. Silicate antibodies in Danish women with silicone breast implants. Rheumatology (Oxford) 2003;42:1032-5.

66. Kossovsky N, Gornbein JA, Zeidler M, et al. Self-reported signs and symptoms in breast implant patients with novel antibodies to silicone surface associated antigens antiSSAA(x). J Appl Biomater 1995;6:153-60.

67. Kossovsky N, Conway D, Kossowsky R, et al. Novel anti-silicone surface-associated antigen antibodies (antiSSAA(x)) may help differentiate symptomatic patients with silicone breast implants from patients with classical rheumatological disease. Curr Top Microbiol Immunol 1996;210:327-36.

68. Smalley DL, Shanklin DR, Hall MF, et al. Immunologic stimulation of $\mathrm{T}$ lymphocytes by silica after use of silicone mammary implants. FASEB J 1995;9:424-7.

69. Shen GQ, Ojo-Amaize EA, Agopian MS, et al. Silicate antibodies in women with silicone breast implants: development of an assay for detection of humoral immunity. Clin Diagn Lab Immunol 1996;3:162-6.

70. Weinzweig J, Schnur PL, McConnell JP, et al. Silicon analysis of breast and capsular tissue from patients with saline or silicone gel breast implants: II. Correlation with connective-tissue disease. Plast Reconstr Surg 1998;101:1836-41.

71. O'Hanlon T, Koneru B, Bayat E, et al. Immunogenetic differences between Caucasian women with and those without silicone implants in whom myositis develops. Arthritis Rheum 2004;50:3646-50.

72. Young VL, Nemecek JR, Schwartz BD, et al. HLA typing in women with breast implants. Plast Reconstr Surg 1995;96:1497-519; discussion 1520.

73. Melmed EP. A review of explantation in 240 symptomatic women: a description of explantation and capsulectomy with reconstruction using a periareolar technique. Plast Reconstr Surg 1998;101:1364-73.

74. Cuellar ML, Gluck O, Molina JF, et al. Silicone breast implant--associated musculoskeletal manifestations. Clin 
Rheumatol 1995;14:667-72.

75. Peters $W$, Smith D, Fornasier V, et al. An outcome analysis of 100 women after explantation of silicone gel breast implants. Ann Plast Surg 1997;39:9-19.

76. Svahn JK, Vastine VL, Landon BN, et al. Outcome of mammary prostheses explantation: a patient perspective. Ann Plast Surg 1996;36:594-600.

77. Colaris MJL, de Boer M, van der Hulst RR, et al. Two hundreds cases of ASIA syndrome following silicone implants: a comparative study of 30 years and a review of current literature. Immunol Res 2017;65:120-8.

78. Maijers MC, de Blok CJ, Niessen FB, et al. Women with silicone breast implants and unexplained systemic symptoms: a descriptive cohort study. Neth J Med 2013;71:534-40.

79. Rohrich RJ, Kenkel JM, Adams WP, et al. A prospective analysis of patients undergoing silicone breast implant explantation. Plast Reconstr Surg 2000;105:2529-37; discussion 2538-43.

80. Godfrey PM, Godfrey NV. Response of locoregional and systemic symptoms to breast implant replacement with autologous tissues: experience in 37 consecutive patients. Plast Reconstr Surg 1996;97:110-6.

81. Solomon G. A clinical and laboratory profile of symptomatic women with silicone breast implants. Semin

Cite this article as: Hoa S, Milord K, Hudson M, Nicolaidis SC, Bourré-Tessier J. Risk of rheumatic disease in breast implant users: a qualitative systematic review. Gland Surg 2021;10(8):2557-2576. doi: 10.21037/gs-21-266
Arthritis Rheum 1994;24:29-37.

82. Kappel RM, Pruijn GJ. The monobloc hydrogel breast implant, experiences and ideas. Eur J Plast Surg 2012;35:229-33.

83. Vasey FB, Havice DL, Bocanegra TS, et al. Clinical findings in symptomatic women with silicone breast implants. Semin Arthritis Rheum 1994;24:22-8.

84. Wallace DJ, Basbug E, Schwartz E, et al. A Comparison of Systemic Lupus Erythematosus and Scleroderma Patients with and without Silicone Breast Implants. J Clin Rheumatol 1996;2:257-61.

85. Thomas WO 3rd, Harper LL, Wong SW, et al. Explantation of silicone breast implants. Am Surg 1997;63:421-9.

86. de Boer M, Colaris M, van der Hulst RRWJ, et al. Is explantation of silicone breast implants useful in patients with complaints? Immunol Res 2017;65:25-36.

87. Hill AB. The environment and disease: association or causation? Proc R Soc Med 1965;58:295-300.

88. Karlson EW, Lee IM, Cook NR, et al. Comparison of self-reported diagnosis of connective tissue disease with medical records in female health professionals: the Women's Health Cohort Study. Am J Epidemiol 1999;150:652-60. 